\title{
A geometric interpretation of zonostrophic instability
}

\author{
Georgios Kontogiannis \\ Laboratory of Meteorology and Climatology, Department of Physics, University of Ioannina, Ioannina, Greece \\ Nikolaos A. Bakas* \\ Laboratory of Meteorology and Climatology, Department of Physics, University of Ioannina, \\ Ioannina, Greece
}

${ }^{*}$ Corresponding author address: Nikolaos Bakas, F2-316, Department of Physics, University of Ioannina, 45110 Ioannina, Greece.

E-mail: nbakas@uoi.gr 


\begin{abstract}
The zonostrophic instability that leads to the emergence of zonal jets in barotropic beta-plane turbulence is analyzed through a geometric decomposition of the eddy stress tensor. The stress tensor is visualized by an eddy variance ellipse whose characteristics are related to eddy properties. The tilt of the ellipse principal axis is the tilt of the eddies with respect to the shear, the eccentricity of the ellipse is related to the eddy anisotropy, while its size is related to the eddy kinetic energy. Changes of these characteristics are directly related to the vorticity fluxes forcing the mean flow. The statistical state dynamics of the turbulent flow closed at second order is employed as it provides an analytic expression for both the zonostrophic instability and the stress tensor. For the linear phase of the instability, the stress tensor is analytically calculated at the stability boundary. For the non-linear equilibration of the instability the tensor is calculated in the limit of small supercriticality in which the amplitude of the jet velocity follows Ginzburg-Landau dynamics. It is found that dependent on the characteristics of the forcing, the jet is accelerated either because the jet primarily anisotropizes the eddies so as to produce upgradient fluxes or because the jet changes the eddy tilt. The instability equilibrates as these changes are partially reversed by the non-linear jet-eddy dynamics.
\end{abstract}




\section{Introduction}

The interaction of small-scale turbulent eddies with large-scale motions in the ocean significantly influences the ocean circulation in many ways (Danabasoglu et al. 1994; Marshall and Speer 2012). In the Southern Ocean, the eddies undertake most of the poleward heat transfer (Walkden et al. 2008) and have an order one effect in the dynamics of the Antarctic Circumpolar Current (ACC) (Danabasoglu et al. 1994; Marshall and Speer 2012) as well as in setting the mean stratification (Cessi and Fantini 2004). In the Western Boundary Currents, the momentum transfer by the eddies maintains the mean jet and significantly influences its structure (Waterman and Hoskins 2013) as well as produces significant jet variability (Qiu 2000). Consequently, there is great interest in studying these interactions. The goal is twofold. The first is understanding the physical mechanisms and dynamics setting the mean circulation, stratification, and transport in the ocean. The second is that the ocean components of climate models do not routinely resolve the mesoscale eddies even in the mid-latitudes where the dominant eddy scale is large. Therefore, the effect of the eddies needs to be parameterized and future climate projections critically depend on the skill of such parameterizations.

Marshall et al. (2012) developed a framework for studying and parameterizing the effect of the small-scale eddies on the large scale flow, a framework that was recently termed GEOMETRIC (Geometry and Energetics of Ocean Mesoscale Eddies and Their Rectified Impact on Climate) by David Marshall and collaborators. The core of this approach is that the eddy forcing of the mean flow is determined by the divergence of the Eliassen-Palm flux tensor. This tensor has a Reynolds stress component that can be visualized by a hyperboloid and an eddy form stress tensor that can be visualized by an ellipsoid. A more concise representation of the Reynolds stress geometry can be obtained by considering a horizontal section of the hyperboloid resulting in the horizontal eddy 
variance ellipse already utilized in barotropic studies (Hoskins et al. 1983). Similarly, a section of the ellipsoid with the vertical plane along the major axis of the ellispoid results in a vertical eddy variance ellipse that represents the geometry of the eddy form stress tensor more compactly (Poulsen et al. 2019). The size and the eccentricity of the ellipses are directly related to the amplitude of the eddy kinetic energy and the eddy anisotropy respectively, while the orientation of the ellipses with respect to the background shear indicates whether the eddies extract energy from the mean flow or surrender their energy to the mean flow.

Viewing the eddy-mean flow interactions through the Eliassen-Palm flux tensor offers three main advantages. The first advantage is that variance ellipses can be directly obtained from observations through principal component analysis of the eddy velocity covariance tensor (Preisendorfer 1988). The variance ellipses have been used to deduce eddy statistics and properties from observations (Morrow et al. 1994; Trani et al. 2011), to compare observational and model surface eddy variability and isotropy (Wilkin and Morrow 1994; Scott et al. 2008), and to analyze eddy anisotropy and its dependence on bathymetry in ocean models (Stewart et al. 2015).

The second advantage is that the tensor can be utilized to diagnose and analyze the eddy-mean flow interactions. Waterman and Hoskins (2013) and Waterman and Lilly (2015) considered a simplified model of a western boundary current extension jet and calculated the statistics of the eddy geometry. They showed that the variance ellipse patterns agreed with predictions of the jet instability and that both the angle and anisotropy of the eddies are important for the evolution of the mean flow. Youngs et al. (2017) utilized the GEOMETRIC framework to diagnose the role of barotropic and baroclinic instability in the energy exchange between a standing meander and a zonal jet in a channel model of the Antarctic Circumpolar Current and recently Poulsen et al. (2019) deduced the vertical ellipses and the eddy form stress in the ACC from model simulations in realistic configurations. 
The third advantage is that the tensor has properties that make parameterization efforts dynamically constrained and more tractable. The reasons are first of all that parameterizations based on this tensor conserve momentum by construction. This should be contrasted to other attempts, as for example downgradient closures of potential vorticity that may not satisfy momentum constraints and require energetic constraints of the mixing efficiency to yield realistic flows (Marshall and Adcroft 2010). Additionally, with the energy specifying the radius of the variance ellipses the resulting tensor is written in terms of five bounded dimensionless parameters that are related to the geometry of the variance ellipses such as their eccentricity and their orientation. The goal is then to develop parameterizations of these ellipse characteristics and it is anticipated that the bounds on these parameters makes the parameterization efforts more tractable. While a simplified version of such a parameterization has been recently implemented with encouraging results (Mak et al. 2017, 2018), further research is needed in linking eddy-mean flow feedbacks to the characteristics of the variance ellipses and in constraining and parameterizing the relevant parameters in simple examples.

In that vein, Marshall et al. (2012) applied the framework to the Eady model (Eady 1949) and showed that if the ellipse tilt is consistent with eddy growth, i.e leaning against the shear, then the framework yields a correct order of magnitude for the growth rate based on dimensional grounds only. Tamarin et al. (2016) applied the framework to barotropic instability and obtained analytic solutions relating the eddy ellipse geometry with the unstable modes. In this work we seek to extend these efforts to the simplified model of forced-dissipative beta-plane turbulence.

In this model, small-scale turbulence supported by random stirring self-organizes in large-scale structures such as zonal jets (Vallis and Maltrud 1993) and large scale waves that remain phase coherent over long time scales and can even exhibit non-dispersive characteristics (Galperin et al. 2010). The jets and the waves were shown to emerge in the flow as symmetry-breaking bifurca- 
tions (Srinivasan and Young 2012; Bakas and Ioannou 2013a; Constantinou et al. 2014a). That is, as the energy input rate of the forcing crosses a critical threshold value, the flow transitions from a homogeneous turbulent state to an inhomogeneous state with the spontaneous emergence of jets or large-scale waves.

The emergence of large-scale structures as a bifurcation, is the result of the cooperative interaction between the small-scale turbulence and the emergent flows giving rise to a collective type of instability (Farrell and Ioannou 2007). This collective type of instability involves the small residual of the statistical mean of the turbulent Reynolds stresses that influences the mean flow coherently, which then modifies the statistics of the distribution of the turbulent eddies to reinforce itself. Therefore a framework addressing the dynamics of the flow statistics is required to analytically express the instability. This statistical state dynamics (SSD) is tractable only with a closure assumption, as a straightforward calculation leads to an infinite hierarchy of equations for the moments (Hopf 1952). A large number of studies in the literature on diverse physical problems ranging from quasi-geostrophic (DelSole 2004; Farrell and Ioannou 2008, 2009a; Marston 2010) and stratified turbulence (Fitzgerald and Farrell 2018a,b) to turbulence in astrophysical flows (Farrell and Ioannou 2009b; Tobias et al. 2011; Parker and Krommes 2013; Constantinou and Parker 2018) and in pipe flows (Constantinou et al. 2014b; Farrell et al. 2017) have shown that a second-order closure of the SSD is accurate in capturing the characteristics and dynamics of the dominant largescale structures. Such closures of the SSD are either referred to as stochastic structural stability theory (S3T) (Farrell and Ioannou 2003) or second-order cumulant expansion (CE2) (Marston et al. 2008).

The collective flow-forming instability was studied within the S3T framework by Farrell and Ioannou (2007) and by Srinivasan and Young (2012); the latter termed the instability as zonostrophic. In these studies, the stability thresholds were calculated along with the characteristics 
of the unstable modes. Constantinou et al. (2014a) and Bakas and Ioannou (2014) compared the instability predictions to direct numerical simulations and showed that they are accurate as long as the dynamics and effect of large-scale waves is taken into account. The equilibration of zonostrophic instability at parameter values just above the stability threshold, was studied by Parker and Krommes (2014) who showed that the velocity amplitude of the emerging jets follows GinzburgLandau (G-L) dynamics. In addition, the quantitative accuracy of the $\mathrm{G}-\mathrm{L}$ approximation was examined by comparison with jet equilibria obtained from the fully nonlinear S3T dynamics (Parker and Krommes 2014; Bakas et al. 2019).

In this work, we study the zonostrophic instability within the GEOMETRIC framework and provide a direct link between the predictions of the S3T dynamics and the characteristics of the eddy variance ellipses. Our goal is to elucidate the relation between the ellipse characteristics and the eddy-mean flow feedbacks in a simple model in which the jets form and are maintained by the turbulent eddies. Jet emergence in barotropic beta-plane turbulence presents the perfect example for this due to two reasons. The first is that the SSD is deterministic and provides a noise-free expression of the ellipse characteristics that in the case of jet emergence are also amenable to analytic treatment. While the eddy-mean flow feedbacks underlying the instability and its equilibration have been previously studied (Bakas and Ioannou 2013b; Bakas et al. 2015, 2019), we find that treating jet formation in terms of the variance ellipses sheds new light into the instability and its equilibration. The second is that zonostrophic instability is markedly different from hydrodynamic instability, in which the perturbations grow in a fixed mean flow. In the flow-forming instability, both the coherent mean flow and the incoherent eddy field are allowed to change. The instability manifests as a weak zonal flow that is inserted in an otherwise homogeneous turbulent field, organizes the incoherent fluctuations to coherently reinforce the zonal flow. Such a collective type of behavior and support also plays a role in maintaining the large-scale flows in the atmosphere and 
the ocean, but has never been treated within the GEOMETRIC framework. Here, we undertake this task and investigate the eddy-mean flow feedbacks in this collective type of instability and further discuss possible parameterizations of the ellipse characteristics.

This paper is organized as follows. In section 2 we present the SSD of barotropic beta-plane turbulence as well as the zonostrophic instability that forms zonal jets in the turbulent flow. In section 3 we review the GEOMETRIC framework for barotropic flows and discuss how changes in the characteristics of the eddy variance ellipse force the zonal flow. In section 4 we calculate the variance ellipse for the unstable jets and relate the change of its characteristics to the upgradient fluxes. Finally, in section 5 we calculate the variance ellipse during the evolution of the instability in the limit of small supercriticality and discuss the eddy-mean flow feedbacks underlying its equilibration. We end with the conclusions in section 6 .

\section{Statistical state dynamics of barotropic $\beta$-plane turbulence and zonostrophic instability}

Consider a forced-dissipative barotropic flow on a $\beta$-plane. The dynamics is governed by the non-linear equation for the evolution of relative vorticity $\tilde{\zeta}$ :

$$
\partial_{\tilde{t}} \tilde{\zeta}+J(\tilde{\psi}, \tilde{\zeta})+\tilde{\beta} \partial_{\tilde{x}} \tilde{\psi}=-r \tilde{\zeta}+\sqrt{\tilde{\varepsilon}} \tilde{\xi}
$$

where $\tilde{\beta}$ is the planetary vorticity gradient, $\tilde{\psi}$ is the streamfunction that is linearly related to vorticity through the inverse of the Laplacian $\Delta=\partial_{\tilde{x}}^{2}+\partial_{\tilde{y}}^{2}\left(\tilde{\psi}=\Delta^{-1} \tilde{\zeta}\right)$ and $J(f, g)=\partial_{\tilde{x}} f \partial_{\tilde{y}} g-$ $\partial_{\tilde{y}} f \partial_{\tilde{x}} g$ is the Jacobian. Bottom drag is parameterized through linear dissipation of vorticity at a rate $r$. The random stirring $\tilde{\xi}$ parameterizes processes such as small-scale convection or baroclinic instability and maintains the turbulence in the flow. It is assumed to be temporally uncorrelated and spatially homogeneous with a prescribed spatial correlation function $Q$ and to inject energy at

a rate $\tilde{\varepsilon}$ in the flow. That is, the correlation of $\tilde{\xi}$ between two different points in space $\left(\tilde{\mathbf{x}}_{a}, \tilde{\mathbf{x}}_{b}\right)$ and 
two different points in time $\left(\tilde{t}_{a}, \tilde{t}_{b}\right)$ is:

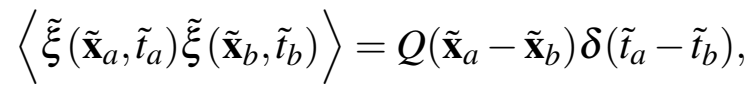

where the brackets denote an average over the realizations of the forcing. In this work we consider an excitation that injects energy in a delta ring in wavenumber space with radius $k_{f}$. That is, the power spectrum of the correlation function is:

$$
\begin{aligned}
\hat{Q}(\tilde{\mathbf{k}}) & =\frac{1}{2 \pi} \int Q\left(\tilde{\mathbf{x}}_{a}-\tilde{\mathbf{x}}_{b}\right) e^{-i \tilde{\mathbf{k}} \cdot\left(\tilde{\mathbf{x}}_{a}-\tilde{\mathbf{x}}_{b}\right)} d^{2} \tilde{\mathbf{x}} \\
& =2 \pi k_{f} \boldsymbol{\delta}\left(\tilde{k}-k_{f}\right)[1+\eta \cos (2 \phi)],
\end{aligned}
$$

where $\tilde{k}$ is the amplitude of the wavevector $\tilde{\mathbf{k}}=\left(\tilde{k}_{x}, \tilde{k}_{y}\right)$ and $\phi=\arctan \left(\tilde{k}_{y} / \tilde{k}_{x}\right)$. We consider two cases of forcing that have been used in previous studies of $\beta$-plane turbulence. An isotropic forcing $(\eta=0)$ that is thought as parametrizing vorticity sources such as convection (Lilly 1969; Vallis and Maltrud 1993; Galperin et al. 2010) and an anisotropic forcing $(\eta=1)$ that injects more power in waves with small $\left|\tilde{k}_{y}\right|$, as if the vorticity injection was due to baroclinic growth processes (Srinivasan and Young 2014; Bakas et al. 2015). We non-dimensionalize equation (1) using the forcing length scale $k_{f}^{-1}$ as the unit of length and the dissipation time scale $r^{-1}$ as the unit of time. The vorticity, the streamfunction, the planetary vorticity gradient and the energy input rate are non-dimensionalized by $\zeta=\tilde{\zeta} / r, \psi=\tilde{\psi} r / k_{f}^{2}, \beta=\tilde{\beta} / k_{f} r$ and $\varepsilon=\tilde{\varepsilon} k_{f}^{2} / r^{3}$ respectively. Therefore the non-dimensional versions of (1) and (3) lack the tildes and have $r=1$ and $k_{f}=1$.

To investigate the eddy-mean flow feedback in the zonostrophic instability and to obtain a clear view of the eddy variance ellipse, we formulate the equations that evolve the flow statistics (SSD). We briefly discuss the derivation of the SSD for the barotropic dynamics, which can be found in previous studies like Farrell and Ioannou (2003) and Srinivasan and Young (2012). Typically one separates the vorticity field into a zonal mean, denoted by the overbar, and a non-zonal eddy 
component, denoted by primes:

$$
\zeta=\bar{\zeta}+\zeta^{\prime}
$$

Substituting (4) in the non-dimensional form of (1), we readily obtain the equations for the evolution of the zonal jet and the non-zonal eddies:

$$
\begin{aligned}
\partial_{t} \bar{u} & =\overline{v^{\prime} \zeta^{\prime}}-\bar{u} \\
\partial_{t} \zeta^{\prime} & =\mathscr{A} \zeta^{\prime}+\underbrace{\overline{J\left(\psi^{\prime}, \zeta^{\prime}\right)}-J\left(\psi^{\prime}, \zeta^{\prime}\right)}_{\text {eddy }-e d d y}+\sqrt{\varepsilon} \xi,
\end{aligned}
$$

where the zonal mean zonal velocity $\bar{u}$ is related to the mean vorticity by $\bar{\zeta}=-\partial_{y} \bar{u}$ and

$$
\mathscr{A}=-\bar{u} \partial_{x}-\left(\beta-\partial_{y}^{2} \bar{u}\right) \partial_{x} \Delta^{-1}-1
$$

is the operator governing the quasi-linear jet-eddy interaction. Under a quasi-linear assumption in which the eddy-eddy interactions noted in (6) are ignored, the SSD of (5)-(6) closes at second order and comprises of equations for the evolution of the first cumulant $\bar{u}(y, t)$, which is the zonal jet velocity and the second cumulant $C\left(\mathbf{x}_{a}, \mathbf{x}_{b}, t\right)=\overline{\zeta^{\prime}\left(\mathbf{x}_{a}, t\right) \zeta^{\prime}\left(\mathbf{x}_{b}, t\right)}$, which is the eddy vorticity correlation. The equations governing the evolution of the two cumulants are ${ }^{1}$ :

$$
\begin{aligned}
\partial_{t} \bar{u} & =\mathscr{R}(C)-\bar{u}, \\
\partial_{t} C & =\left(\mathscr{A}_{a}+\mathscr{A}_{b}\right) C+\varepsilon Q,
\end{aligned}
$$

where the linear operator

$$
\mathscr{R}(C)=\frac{1}{2}\left[\left(\partial_{x_{a}} \Delta_{a}^{-1}+\partial_{x_{b}} \Delta_{b}^{-1}\right) C\right]_{a=b},
$$

gives the vorticity flux as a function of the eddy vorticity correlation $C$. The subscripts in the operators denote differentiation with respect to the different points $\mathbf{x}_{a}$ and $\mathbf{x}_{b}$ as well as evaluation

\footnotetext{
${ }^{1}$ Details on the derivation of the SSD can be found in previous studies, e.g. Farrell and Ioannou (2003); Srinivasan and Young (2012); Bakas et al. (2015).
} 
of the mean velocity at these points. In (10), the subscript $a=b$ denotes the evaluation of the expression in the brackets at the same point. The closed deterministic system (8)-(9), termed as S3T dynamics, comprises the joint evolution of the jet and its associated eddy statistics.

For all values of $\beta$ and $\varepsilon$, the state with zero mean flow and a homogeneous covariance proportional to the forcing covariance:

$$
\bar{u}_{0}=0, C_{0}\left(\mathbf{x}_{a}-\mathbf{x}_{b}\right)=\frac{\varepsilon}{2} Q\left(\mathbf{x}_{a}-\mathbf{x}_{b}\right),
$$

is a fixed point of the S3T dynamics. Jets emerge in the flow as this homogeneous equilibrium becomes unstable when the energy input rate passes a critical value (Farrell and Ioannou 2007). The incipient instability that is termed zonostrophic, is addressed by linearizing (8)-(9) around equilibrium (11). The eigenfunctions consist of a harmonic jet $\delta \bar{u}=e^{i n y}$ with wavenumber $n$ and a corresponding eddy covariance that is given by:

$$
\delta C=\left[G^{+}\left(\mathbf{x}_{a}-\mathbf{x}_{b}\right)-G^{-}\left(\mathbf{x}_{a}-\mathbf{x}_{b}\right)\right] e^{i n\left(y_{a}+y_{b}\right) / 2},
$$

where

$$
G^{ \pm}(\mathbf{x})=\int \frac{d^{2} \mathbf{k}}{2 \pi} \frac{i k_{x} k_{\mp}^{2}\left(k_{ \pm}^{2}-n^{2}\right)}{(\sigma+2) k_{+}^{2} k_{-}^{2}+2 i \beta n k_{x} k_{y}} \frac{\hat{Q}\left(\mathbf{k}_{ \pm}\right)}{2} e^{i \mathbf{k} \cdot \mathbf{x}},
$$

$\mathbf{n}=(0, n), \mathbf{k}_{ \pm}=\mathbf{k}+\mathbf{n} / 2$ and $k_{ \pm}=\left|\mathbf{k}_{ \pm}\right|$. The dispersion relation for the eigenvalues $\sigma$ was shown by Bakas et al. (2015) to be:

$$
\sigma=\varepsilon f(\sigma \mid \delta \bar{u}, Q / 2)-1
$$

The term $f$ is given in (A1) and expresses the vorticity flux induced by the covariance perturbation $\delta C$. The vorticity flux $f$ as well as the eigenvalues are real in this case for jet perturbations with scales larger than the forcing scale $(n<1)$. A jet with wavenumber $n$ is therefore rendered unstable under two conditions: (i) the vorticity fluxes $f$ are positive, that is the distortion of the eddies by the jet as expressed by $\delta C$ produces upgradient fluxes and (ii) the amplitude of the fluxes should 
be large enough to overcome dissipation. This second condition is satisfied when the energy input rate is above a critical value $\varepsilon_{t}(n)$ that can be computed from (14) by assuming criticality $(\sigma=0)$ : $\varepsilon_{t}=1 / f(0 \mid \delta \bar{u}, Q / 2)$. The minimum value of $\varepsilon_{t}$ over all wavenumbers

$$
\varepsilon_{c}=\min _{n}\left[\varepsilon_{t}(n)\right]
$$

is the stability boundary above which jets form. The critical rate $\varepsilon_{c}$ is attained by the most unstable jet with wavenumber $n_{c}$. Details on the characteristics of the dispersion relation of the zonostrophic instability and the stability boundary (15) can be found in Srinivasan and Young (2012) and Bakas et al. (2015). The goal in this work is to investigate the eddy fluxes producing the instability through analysis of the eddy variance ellipse and to illuminate the jet-eddy interactions leading to jet formation.

\section{The GEOMETRIC approach and the eddy variance ellipse}

In this section we review the GEOMETRIC approach as is applied for barotropic flows and closely follow the discussion and notation in Tamarin et al. (2016). The GEOMETRIC approach relies in expressing the vorticity fluxes that force the mean flow in terms of the eddy momentum stress tensor $\mathbf{T}$ through the Taylor identity:

$$
\overline{\mathbf{u}^{\prime} \zeta^{\prime}}=\nabla \cdot \mathbf{T}
$$

where $\mathbf{T}$ is for the barotropic dynamics considered (Tamarin et al. 2016):

$$
\mathbf{T}=\left(\begin{array}{cc}
N & M \\
M & -N
\end{array}\right),
$$

with $N=\overline{u^{\prime} v^{\prime}}$ the eddy momentum fluxes and $M=(1 / 2)\left(\overline{v^{\prime 2}}-\overline{u^{\prime 2}}\right)$ a quantity measuring the anisotropy of the eddies. One of the advantages of expressing the vorticity fluxes in terms of the tensor $\mathbf{T}$ is that the stress tensor can be visualized geometrically through an eddy variance ellipse, 
the characteristics of which (tilt, eccentricity and focal point) can be obtained at each point in the flow. Therefore, by examining how the ellipse changes throughout the flow we can deduce, through expression (16), the mean flow forcing.

The geometric representation of the ellipse becomes evident be rewriting the tensor as (Marshall et al. 2012; Tamarin et al. 2016):

$$
\mathbf{T}=\gamma K\left(\begin{array}{cc}
\sin 2 \theta & -\cos 2 \theta \\
-\cos 2 \theta & -\sin 2 \theta
\end{array}\right),
$$

where $K=(1 / 2)\left(\overline{u^{\prime 2}}+\overline{v^{\prime 2}}\right)$ is the eddy kinetic energy, $\theta=(1 / 2) \arctan (-N / M)$ is the tilt of the ellipse principal axis and $\gamma=\sqrt{N^{2}+M^{2}} / K$ is a non-dimensional parameter related to the eddy anisotropy that was shown by Marshall et al. (2012) to be bounded ( $0 \leq \gamma \leq 1)$. The eccentricity $e$ of the ellipse is determined by the anisotropy parameter as $e=\sqrt{2 \gamma /(1+\gamma)}$. The size of the ellipse is determined by the kinetic energy and the anisotropy parameter, as the linear eccentricity (the distance $F$ between a focal point and the center of the ellipse) is $F=2 \sqrt{\gamma K}$. Therefore, the semi-major axis of the ellipse is $a=F / e=\sqrt{2 K(1+\gamma)}$. The ellipse in the eddy velocity space and its characteristics are visualized in Fig. 1.

In order to relate the eddy fluxes that force the mean flow to the spatial changes in the ellipse characteristics $(\gamma, \theta, K)$ we substitute T from (18) into Taylor's identity (16) and obtain:

$$
\overline{v^{\prime} \zeta^{\prime}}=-\left(\gamma \frac{\partial K}{\partial y}+K \frac{\partial \gamma}{\partial y}\right) \sin 2 \theta-2 \gamma K \frac{\partial \theta}{\partial y} \cos 2 \theta
$$

Therefore, the meridional gradients of the three parameters (the three terms in equation (19)) can lead to a jet acceleration or deceleration depending on both the sign of the gradient and the orientation of the ellipse. Figure 2 shows the different cases that illustrate how the three gradients influence the sign of the vorticity fluxes. For example, we see that if the eddy kinetic energy (column B) or if the eccentricity (column D) increase with latitude and the ellipse tilt angle is in 
the second (or fourth) quadrant, then this leads to an eastward acceleration of the zonal flow. An eastward acceleration also occurs if, for example, the axial tilt is $|\theta| \leq \pi / 4$ and decreases with latitude (column E). Note that opposite changes to the ones shown in the columns of Fig. 2, imply westward acceleration. Our goal is to investigate the eddy variance ellipse characteristics at the onset of zonostrophic instability and the contribution of the changes of the three characteristics to the positive vorticity flux feedback that underlies the instability.

\section{The eddy variance ellipse at the onset of zonostrophic instability}

To obtain the eddy variance ellipse at the onset of zonostrophic instability, we calculate the eddy momentum fluxes $N$, the eddy anisotropy $M$ as well as the eddy kinetic energy $K$ for the most unstable jet $\bar{u}_{1}=\sin \left(n_{c} y\right)$ with wavenumber $n_{c}$ at onset $(\sigma=0)$ :

$$
\begin{aligned}
N & =N_{1}=\hat{N}_{1}(\beta) \cos \left(n_{c} y\right), \\
M & =M_{0}+M_{1}=M_{0}+\hat{M}_{1}(\beta) \sin \left(n_{c} y\right), \\
K & =K_{0}+K_{1}=K_{0}+\hat{K}_{1}(\beta) \sin \left(n_{c} y\right) .
\end{aligned}
$$

The subscript 0 denotes the quantities calculated for the homogeneous equilibrium (11). The subscript 1 denotes the small perturbations induced by the jet $\bar{u}_{1}$ and whose amplitudes depend on the planetary vorticity gradient $\beta$. Note that for the homogeneous equilibrium the momentum fluxes are zero. We first calculate the stress tensor for the isotropic forcing $(\eta=0)$. In Appendix A we show that in this case, $M_{0}=0$ due to the isotropy of the forced eddies, $K_{0}=\varepsilon_{c} / 2$, and $\hat{N}_{1}, \hat{M}_{1}$, are given by the integrals (A5), (A8). Asymptotic values of the integrals in the limits of small and large $\beta$ are also derived (Eqs. (A13), (A15) and (A29)). Figure 3 shows the amplitudes $\hat{N}_{1}$ and $\hat{M}_{1}$

as a function of $\beta$. We observe that the amplitude of the momentum fluxes $\hat{N}_{1}$ is a monotonically increasing function of $\beta$ reaching the minimum value of $\hat{N}_{1} \simeq 1$ for $\beta \ll 1$ while increasing as 
$\hat{N}_{1} \simeq(2 \beta / 3)^{1 / 3}$ for $\beta \gg 1$. The amplitude of the eddy anisotropy $\hat{M}_{1}$ asymptotically increases as $\hat{M}_{1} \simeq(12 \beta / 270)^{1 / 3}$ for $\beta \ll 1$, it reaches a maximum value for $\beta \sim \mathscr{O}(1)$ and subsequently decreases as $\hat{M}_{1} \simeq\left(3 / 2^{10} \beta\right)^{1 / 3}$ for $\beta \gg 1$.

The variance ellipse characteristics can then be readily derived. The tilt and the anisotropy parameter are to leading order:

$$
\begin{gathered}
\theta_{1}=\frac{1}{2} \arctan \left(-\frac{\hat{N}_{1}}{\hat{M}_{1}} \cot \left(n_{c} y\right)\right), \\
\gamma_{1}=\frac{\sqrt{\hat{N}_{1}^{2} \cos ^{2}\left(n_{c} y\right)+\hat{M}_{1}^{2} \sin ^{2}\left(n_{c} y\right)}}{K_{0}} .
\end{gathered}
$$

Figure 4 shows the tilt and the anisotropy parameter as a function of latitude for a wavelength of the most unstable jet and for two values of $\beta$. The tilt angle for both values of $\beta$ is piecewise constant assuming the values of $\pi / 4$ in the first quarter of the jet wavelength, $3 \pi / 4$ in the second and third quarter of the jet wavelength and $5 \pi / 4$ in the last quarter. The angle increases within a very narrow width of latitudes in the vicinity of the jet cores $\left(y n_{c} \simeq \pi / 2\right.$ and $\left.y n_{c} \simeq 3 \pi / 2\right)$. The reason for the piecewise constant profile of the tilt is that based on the asymptotic scalings (A13) and (A15), the amplitude of the eddy anisotropy is much smaller than the amplitude of the momentum fluxes $\left(\hat{M}_{1} \ll \hat{N}_{1}\right)$ for both low and high values of $\beta$. As a result their ratio is large yielding values that are odd multiples of $\pi / 4$ for the angle $\theta_{1}$. The only latitudes over which the tilt is different, are near the jet core $\left(\left|y n_{c}\right| \sim \pi / 2\right)$ where the momentum fluxes are small as well and $\hat{N}_{1} \cot \left(n_{c} y\right) / \hat{M}_{1} \sim \mathscr{O}(1)$. The reason is that symmetry considerations require an angle of zero or $\pi / 2$ right at the jet core (Tamarin et al. 2016). In contrast, we have significant changes in the anisotropy parameter $\gamma_{1}$ over all latitudes. Since the amplitude of the eddy anisotropy $\left(\hat{M}_{1}\right)$ is much smaller than the amplitude of the momentum fluxes $\left(\hat{N}_{1}\right)$ the anisotropy parameter $\gamma_{1} \propto\left|\cos \left(n_{c} y\right)\right|$. Therefore the eddies are almost isotropic at the jet core where we have minimum values of $\gamma_{1}$ and anisotropic at the regions of maximum shear where we have maximum values of $\gamma_{1}$. 
The change of these parameters leads to concomitant changes of the variance ellipse with latitude that are schematically shown in Fig. 4(c). First of all, we observe that the eddies lean with the jet shear, which is consistent with the intuitive picture of the eddies surrendering their energy to the mean flow. In the first quarter of the jet wavelength, the increase of the tilt and the decrease of the anisotropy parameter with latitude corresponds to patterns $\mathrm{C}$ and $\mathrm{F}$ of Fig. 2, leading to an eastward acceleration of the flow. An eastward acceleration is induced in the second quarter of the wavelength as well, due to the increase of the tilt angle and the anisotropy parameter with latitude with $|\theta|>\pi / 4$ (patterns $\mathrm{D}$ and $\mathrm{F}$ ). In the second half of the jet wavelength, the increase of tilt with latitude corresponds to the opposite pattern of $\mathrm{D}$ and leads to a westward acceleration. Similarly, the increase of the ellipse eccentricity in the third quarter of the wavelength with the tilt angle in the second quadrant and the decrease of the ellipse eccentricity in the last quarter of the jet wavelength with the tilt angle in the third quadrant correspond to the opposite patterns of $\mathrm{D}$ and $\mathrm{C}$ and lead to westward acceleration as well. As a result, both the changes in the tilt and the eccentricity reinforce the jet perturbation.

The contribution of the gradients of each of the variance ellipse parameters in the jet acceleration is given to leading order by (cf. Equation (B23)):

$$
\overline{v^{\prime} \zeta^{\prime}} \simeq f_{1}=-K_{0} \frac{\partial \gamma_{1}}{\partial y} \sin 2 \theta_{1}-2 \gamma_{1} K_{0} \frac{\partial \theta_{1}}{\partial y} \cos 2 \theta_{1}
$$

and is shown in Fig. 5 for $\beta=0.1$ and $\beta=10$. The first term of (19) that involves the kinetic energy gradient is second order with respect to the infinitesimal jet perturbation and is, therefore, negligible. We observe that the jet is intensified due to the gradient of the anisotropy parameter $\gamma_{1}$ for most latitudes, while the gradient of the tilt contributes significantly only at the jet core.

For the anisotropic forcing, the eddy anisotropy and the eddy kinetic energy are to leading order equal to the values calculated for the homogeneous equilibrium $M_{0}=K_{0}=\varepsilon_{c} / 2$, while the eddy 
momentum fluxes are of the same order as the jet perturbation. The amplitude of the fluxes that is shown in Fig. 3(c) is constant $\left(\hat{N}_{1} \simeq \sqrt{2}\right)$ for low values of $\beta$, while for $\beta \gg 1$ it increases as $\hat{N}_{1} \simeq(3 \beta / 4)^{1 / 3}$. The eddy anisotropy parameter is to leading order:

$$
\gamma_{0}=\frac{M_{0}}{K_{0}}=1,
$$

yielding a highly eccentric ellipse with eccentricity slightly smaller than one. The tilt angle

$$
\theta_{1}=\frac{\pi}{2}-\frac{1}{2} \frac{\hat{N}_{1} \cos \left(n_{c} y\right)}{M_{0}}
$$

varies sinusoidally at leading order around $\pi / 2$. This leads to the changes of the ellipse variance with latitude that are schematically shown in Fig. 6. We observe again that the ellipse tilt is consistent with the intuitive picture of the eddies leaning with the jet shear in order to produce upgradient fluxes. The ellipse focal point and eccentricity determined by $M_{0}$ and $K_{0}$ are constant across latitude, while the tilt increases in the first half of the jet wavelength. This corresponds to pattern E of Fig. 2 leading to an eastward acceleration of the flow. In the second half of the jet wavelength, the tilt decreases with latitude and corresponds to the opposite change than pattern E, leading to a westward acceleration of the jet. Therefore the jet perturbation is reinforced. Since the terms of (19) involving the kinetic energy and the anisotropy parameter gradients are of second order with respect to the infinitesimal jet perturbation, the jet is intensified solely due to the gradient of the tilt angle $\theta_{1}$. In this case the vorticity fluxes are to leading order given by (cf. Equation (B33)):

$$
\overline{v^{\prime} \zeta^{\prime}} \simeq f_{1}=2 \gamma_{0} K_{0} \frac{\partial \theta_{1}}{\partial y}=n_{c} \hat{N}_{1} \sin \left(n_{c} y\right)
$$

Since the jet is accelerated by the change of the ellipse tilt, we can parameterize this term. It was shown by Bakas and Ioannou (2013b) that the eddy fluxes act as negative diffusion for $\beta \ll 1$, while in the limit of $\beta \gg 1$ the fluxes act as a constant term proportional to the jet velocity. 
Therefore, changes in the tilt in these limits can be parameterized as proportional to the shear $(\theta \sim$ $\pi / 2-d U / d y)$ and proportional to the integral of the jet velocity $\left(\theta \sim \pi / 2+\int U d y\right)$ respectively.

\section{The eddy variance ellipse during the equilibration of the zonostrophic instability}

In this section we discuss the changes in the variance ellipse that bring about the equilibration of zonostrophic instability. It can be readily shown that for energy input rates slightly above the critical threshold $\varepsilon=\left(1+\mu^{2}\right) \varepsilon_{c}$, where $\mu \ll 1$, zonal jets with wavenumbers $\left|n-n_{c}\right|=\mathscr{O}(\mu)$ grow at a rate $\sigma=\mathscr{O}\left(\mu^{2}\right)$. Guided by this observation, Parker and Krommes (2014) considered that the jet velocity varies on the long time scale $T=\mu^{2} t$ and is given by an expansion in $\mu$ :

$$
\bar{u}=\mu \bar{u}_{1}(y, T)+\mu^{2} \bar{u}(y, T)+\mu^{3} \bar{u}_{3}(y, T)+\mathscr{O}\left(\mu^{4}\right),
$$

with a similar expansion for the slowly varying eddy covariance

$$
C=C_{0}\left(\mathbf{x}_{a}-\mathbf{x}_{b}\right)+\mu C_{1}\left(\mathbf{x}_{a}, \mathbf{x}_{b}, T\right)+\mu^{2} C_{2}\left(\mathbf{x}_{a}, \mathbf{x}_{b}, T\right)+\mu^{3} C_{3}\left(\mathbf{x}_{a}, \mathbf{x}_{b}, T\right)+\mathscr{O}\left(\mu^{4}\right) .
$$

The order one terms yield the homogeneous equilibrium with $C_{0}=\varepsilon_{c} Q / 2$, while the order $\mu$ terms yield the eigenfunction (12) with amplitude $A(T)$ :

$$
\begin{aligned}
& \bar{u}_{1}=A(T) e^{i n_{c} y}+A^{*}(T) e^{-i n_{c} y} \\
& C_{1}=\hat{C}_{1}^{1} e^{i n_{c}\left(y_{a}+y_{b}\right) / 2}+\left(\hat{C}_{1}^{1}\right)^{*} e^{-i n_{c}\left(y_{a}+y_{b}\right) / 2}
\end{aligned}
$$

where $\hat{C}_{1}^{1}=A(T)\left[G^{+}\left(\mathbf{x}_{a}-\mathbf{x}_{b}\right)-G^{-}\left(\mathbf{x}_{a}-\mathbf{x}_{b}\right)\right]$. At order $\mu^{2}$, the quasi-linear interaction of the infinitesimal jet $\bar{u}_{1}$ with the eddy covariance perturbation $C_{1}$ generates a double-harmonic jet with velocity $\bar{u}_{2}=a_{2} A^{2} e^{2 i n_{c} y}+$ c.c, where $a_{2}$ is an order one constant given by (B3) and a corresponding covariance correction

$$
C_{2}=\hat{C}_{2}^{0}+\hat{C}_{2}^{2} e^{2 i n_{c}\left(y_{a}+y_{b}\right) / 2}+\left(\hat{C}_{2}^{2}\right)^{*} e^{-2 i n_{c}\left(y_{a}+y_{b}\right) / 2} .
$$


This contains a double-harmonic component to support the double-harmonic jet through the corresponding vorticity fluxes and a homogeneous component that was shown by Bakas et al. (2019) to be a necessity of energy conservation: as the total energy is conserved, the growing energy of the emerging jet has to be drained from the eddy energy and the homogeneous correction facilitates this exchange.

At the next order, elimination of secular terms yields that the amplitude of the emerging jet satisfies the Ginzburg-Landau equation:

$$
c_{1} \partial_{T} A=A-c_{3}|A|^{2} A,
$$

where the coefficients $c_{1}$ and $c_{3}$ are given by (B11) and (B12) and for the ring forcing considered here, are real and positive. The linear term in (33) drives the zonostrophic instability, while the non-linear term is stabilizing and leads to its equilibration at the amplitude $A=1 / \sqrt{c_{3}}$. It is shown in Bakas et al. (2019) that the non-linear term comprises two parts $c_{3}=c_{3}^{e c}+c_{3}^{12}$. The first part is due to the interaction of the first order jet $\bar{u}_{1}$ with the homogeneous correction to the covariance $\hat{C}_{2}^{0}$ and was referred to as the energy correction term. The second part is due to the interaction of $\bar{u}_{1}$ with the double harmonic $\hat{C}_{2}^{2} e^{2 i n_{c}\left(y_{a}+y_{b}\right) / 2}$ and the interaction of $\bar{u}_{2}$ with $C_{1}$. We can therefore deduce which type of eddy-mean flow interactions underlie the equilibration of the instability.

It is shown in Appendix B that for isotropic forcing, the vorticity fluxes underlying the amplitude tendency in (33) are (cf. Equation (B28)):

$$
\begin{aligned}
f_{3} & =\underbrace{f_{1}-K_{0} \frac{\partial \gamma_{3, N L}}{\partial y} \sin 2 \theta_{1}-2 K_{0} \frac{\partial \gamma_{1}}{\partial y} \theta_{3, N L} \cos 2 \theta_{1}}_{f_{d \gamma}} \\
+ & \underbrace{4 \frac{\partial \theta_{1}}{\partial y} \gamma_{1} \theta_{3, N L} \sin 2 \theta_{1}-2 K_{0}\left(\frac{\partial \theta_{3, N L}}{\partial y} \gamma_{1}+\frac{\partial \theta_{1}}{\partial y} \gamma_{3, N L}\right) \cos 2 \theta_{1}}_{f_{d \theta}} .
\end{aligned}
$$

The first term $f_{1}$, is the flux given by (25) that underlies the linear term in (33) and drives the zonostrophic instability. The other two terms $\left(f_{d \gamma}\right.$ and $\left.f_{d \theta}\right)$ underlie the non-linear stabilizing term 
with $\theta_{3, N L}, \gamma_{3, N L}$ given by (B26) and (B27) respectively. In the limit of $|A| \ll 1$, these terms are $|A|^{2}$ smaller than the first term and (34) is to leading order

$$
\begin{aligned}
f_{3} & \simeq-K_{0}\left[\frac{\partial}{\partial y}\left(\gamma_{1}+\gamma_{3, N L}\right)\right] \sin \left(2 \theta_{1}+2 \theta_{3, N L}\right) \\
& -K_{0}\left(\gamma_{1}+\gamma_{3, N L}\right)\left[\frac{\partial}{\partial y} \sin \left(2 \theta_{1}+2 \theta_{3, N L}\right)\right] .
\end{aligned}
$$

Therefore in this limit, $\theta_{3, N L}$ and $\gamma_{3, N L}$ are the non-linear corrections to the tilt angle and the eddy anisotropy parameter respectively. These corrections are shown in Fig. 7 and Fig. 8 for $\beta=0.1$ and $\beta=10$ respectively. For the low value of $\beta$ (Fig. 7(a)-(b)), we observe that the anisotropy parameter is decreased in the regions of maximum shear thereby decreasing the gradient of $\gamma$. The correction to the tilt angle has a dipole structure reducing the gradient of the tilt at the jet core while increasing it in the regions of maximum shear. The contribution of the gradients of these corrections $\left(f_{d \gamma}\right.$ and $\left.f_{d \theta}\right)$ to the vorticity fluxes are shown in Fig. 7(c). We can see that both $f_{d \gamma}$ and $f_{d \theta}$ oppose the resulting contributions driving the instability and that the correction due to the decrease of anisotropy is dominant except at the jet cores. Therefore in this limit, a partial isotropization of the eddies equilibrates the instability. For the large value of $\beta$ (Fig. 8(a)-(b)), we observe that the gradient of the anisotropy parameter is decreased through an increase of $\gamma$ at the jet cores and a decrease in the regions of maximum shear. The correction to the tilt angle has the same dipole structure as in the low $\beta$ limit. The contribution of the gradients of these corrections to the vorticity fluxes are shown in Fig. 8(c). We can see that both gradients are of the same order and roughly follow the same pattern. As a result, the stabilizing fluxes are the small residual of these two opposing contributions. The contribution of the interaction of the jet $\bar{u}_{1}$ with the homogeneous correction to the eddy covariance (termed as energy correction) and the interaction of the single harmonic jet-eddy components with their double harmonic counterparts to the tilt angle $\left(\theta_{3, N L}\right)$ and the anisotropy parameter $\left(\gamma_{3, N L}\right)$ corrections are given by (B26), (B27) and are 
shown in Figures 7-8. We observe that for low $\beta$ the energy correction interaction determines the angle correction while the interaction of the single and the double harmonic jet-eddy components mostly determines the anisotropy parameter correction. Since $f_{d \gamma}$ is the dominant contribution to the fluxes, the interaction of the single and the double harmonic jet-eddy components underlies the stabilizing fluxes. This should be contrasted to the corresponding contributions in the large $\beta$ limit shown in Fig. 8(a)-(b). We can see that in this limit the energy correction interactions determine both the tilt and the anisotropy parameter corrections and therefore these interactions underlie the stabilizing term.

For anisotropic forcing, the vorticity fluxes underlying the amplitude tendency in (33) are:

$$
f_{3}=f_{1}+2 K_{0} \gamma_{0} \frac{\partial \theta_{3, N L}}{\partial y}
$$

where $f_{1}$ is given by (28) and drives the instability and

$$
\theta_{3, N L}=|A|^{2}\left(\frac{\pi}{2}-\theta_{1}\right)
$$

is the non-linear correction to the tilt angle. Therefore, the gradient of $\theta_{3, N L}$ opposes the gradient of $\theta_{1}$ hindering the instability and at equilibrium it exactly cancels it out. It is also worth noting that since $\theta_{3, N L}$ is proportional to $\theta_{1}$, the parametrization of the angle discussed in the previous section holds for the equilibration phase of the instability as well. The contribution of the energy correction interactions $\left(\theta_{3, e c}\right)$ and the interaction of the single harmonic jet-eddy components with their double harmonic counterparts to the tilt angle $\left(\theta_{3,12}\right)$ are given by (B35). It is found that the energy correction interactions dominate the contribution for all values of $\beta$ (not shown) and as a result these interactions underlie the stabilizing fluxes. 


\section{Conclusion}

We revisited the emergence of zonal jets in forced-dissipative barotropic beta-plane turbulence providing a direct link between the eddy-mean flow dynamics underlying jet formation and the characteristics of the Reynolds stress tensor. This tensor is the central object of the GEOMETRIC approach, a framework for studying and parameterizing the effect of small-scale eddies on the large-scale flow. To address this, we employed the statistical state dynamics of the turbulent flow closed at second order as it provides both an analytic expression for the zonostrophic instability (that was shown by previous studies to form the zonal jets) and an analytic expression for the stress tensor.

Zonostrophic instability arises as the homogeneous statistical equilibrium turbulent state becomes unstable when the energy input rate of the forcing passes a critical value. In order to address the linear phase of the instability, the stress tensor was calculated at the stability boundary, that is when the energy input rate assumes this critical value both for isotropic forcing and for anisotropic forcing mimicking baroclinic instability. The stress tensor, which is visualized by an eddy variance ellipse, was rewritten in terms of two bounded parameters, the ellipse tilt and the anisotropy parameter determining the ellipse eccentricity, as well as the eddy kinetic energy determining the size of the ellipse. The change of the ellipse characteristics were then linked to the eddy vorticity fluxes that form the jets.

We found that for isotropic forcing, changes in the eddy kinetic energy are of higher order and do not influence the emerging jet. Regarding the tilt of the ellipse, we found that the eddies tilt with the shear surrendering their energy to the mean flow as would be intuitively expected for jet formation with the tilt angle being piecewise constant. Therefore, the gradient of the tilt that contributes directly to jet acceleration is very small except at the jet cores. In the bulk of the flow, 
the jet is accelerated as the infinitesimal jet perturbation anisotropizes the eddies in such a way as to produce upgradient fluxes. The anisotropy parameter is maximum at regions of large shear and minimum at the jet core. For anisotropic forcing, both the eddy kinetic energy and the anisotropy parameter are of higher order and the jet is accelerated solely due to changes in the ellipse tilt. The change in the tilt can be parameterized in this case as proportional to the shear for low values of the planetary vorticity gradient or as the integral of the flow for large values of the planetary vorticity gradient, a parametrization that was shown to hold even in the equilibration stage of the instability.

The non-linear phase of zonostrophic instability and its equilibration were then studied in the limit in which the energy input rate is slightly above the critical value for jet formation. In this limit, the amplitude of the jet velocity follows the weakly non-linear Ginzburg-Landau dynamics therefore allowing us to obtain analytic expressions for the stress tensor and its characteristics and to identify the two types of interaction that underlie the equilibration. The first is the interaction of the infinitesimal jet with the homogeneous correction to the eddy covariance that ensures total energy conservation and was termed as energy correction by Bakas et al. (2019). The second is the interaction of the single harmonic jet-eddy components with their double harmonic counterparts that are generated at higher order. For isotropic forcing, the anisotropy parameter is decreased in the regions of maximum shear during the non-linear phase thereby isotropizing the eddies. For low values of the planetary vorticity gradient this is achieved by the interaction of the single harmonic jet-eddy components with their double harmonic counterparts, while for large values of the planetary vorticity gradient this is achieved by the energy correction interactions. The energy correction interactions decrease the gradient of the tilt angle at the jet core and increase it in the regions of maximum shear. For low values of the planetary vorticity gradient, the instability equilibrates due to the partial isotropization of the eddies, while for large values of the planetary 
vorticity gradient the jet equilibrates at its finite amplitude due to opposing gradients from both of the tilt and the anisotropy parameter. For anisotropic forcing, the energy correction interactions simply reduce the tilt changes of the linear phase of the instability and equilibrate the flow. In summary, the geometric decomposition of the stress tensor in zonostrophic instability showed that either the anisotropization of the eddies (for isotropic forcing) or the change of the eddy tilt (for anisotropic forcing) drive the emerging jet and that the instability equilibrates as these changes are partially reversed by the non-linear jet-eddy dynamics.

Acknowledgments. The authors would like to thank Navid Constantinou for helpful comments on the manuscript.

\section{APPENDIX A}

\section{Calculation of the stress tensor at the onset of zonostrophic instability}

In this Appendix we calculate the stress tensor at the onset of zonostrophic instability. We first calculate the most unstable mode with wavenumber $n_{c}$ yielding the maximum vorticity fluxes and the critical energy input rate $\varepsilon_{c}$ above which this mode becomes unstable and jets form. The vorticity fluxes induced by the eigenfunction (12) are (Srinivasan and Young 2012; Bakas et al. 2015):

$$
\begin{aligned}
& \overline{v^{\prime} \zeta^{\prime}}=\mathscr{R}(\delta C)=\varepsilon f(\sigma \mid \delta \bar{u}, Q / 2) e^{i n y} \\
= & \varepsilon e^{i n y} \int \frac{d^{2} \mathbf{k}}{2 \pi} \frac{n k_{x}^{2}\left(k_{y}+n / 2\right)\left(1-n^{2} / k^{2}\right)}{(\sigma+2) k^{2} k_{s}^{2}+2 i \beta n k_{x}\left(k_{y}+n / 2\right)} \hat{Q}(\mathbf{k}),
\end{aligned}
$$

with $k_{s}=|\mathbf{k}+\mathbf{n}|$. In order to calculate $n_{c}$, $\varepsilon_{c}$, we assume criticality $(\sigma=0)$, substitute the ring forcing power spectrum (3), express the integrand in polar coordinates $\left(k_{x}, k_{y}\right)=(k \cos \phi, k \sin \phi)$ and integrate over $k$ to obtain:

$$
\hat{f}_{1}=\int_{0}^{2 \pi} \frac{N_{f}}{D_{f}+i \beta D_{\beta}} d \phi,
$$


with $N_{f}=n \cos ^{2} \phi(\sin \phi+n / 2)\left(1-n^{2}\right)[1+\eta \cos (2 \phi)] / 2 \pi, D_{f}=1+2 n \sin \phi+n^{2}$ and $D_{\beta}=$ $n \cos \phi(\sin \phi+n / 2)$. By numerically calculating the integral and maximizing its value over $n$, we identify the most unstable jet with wavenumber $n_{c}$. The energy input rate $\varepsilon_{c}$ is then given by (15). The integral is real with $\hat{f}_{1}(-n)=\hat{f}(n)$. Therefore, for the sinusoidal jet $\bar{u}_{1}=\sin \left(n_{c} y\right)$ the vorticity fluxes at the onset are:

$$
\overline{v^{\prime} \zeta^{\prime}}=\operatorname{Im}\left(\varepsilon_{c} \hat{f}_{1}\left(n_{c}\right) e^{i n_{c} y}\right)=\varepsilon_{c} \hat{f}_{1}\left(n_{c}\right) \sin \left(n_{c} y\right)
$$

We then calculate the elements $N$ and $M$ of the stress tensor as well as the eddy kinetic energy $K$. The momentum fluxes $N$ are given by the action of the operator $\mathscr{N}$ on the covariance:

$$
\begin{aligned}
N & =\mathscr{N}\left(C_{0}+\delta C\right) \\
& \stackrel{\text { def }}{=}-\frac{1}{2}\left[\left(\partial_{x_{a}} \partial_{y_{b}}+\partial_{x_{b}} \partial_{y_{a}}\right) \Delta_{a}^{-1} \Delta_{b}^{-1}\left(C_{0}+\delta C\right)\right]_{a=b} .
\end{aligned}
$$

After substitution of the eigenfunction (12), we find that the induced fluxes for the sinusoidal jet perturbation $\bar{u}_{1}$ are:

$$
N=\operatorname{Im}\left[\frac{i \varepsilon}{n} f(\sigma \mid \delta \bar{u}, Q / 2) e^{i n y}\right]=\hat{N}_{1} \cos (n y)
$$

where $\hat{N}_{1}=\hat{f}_{1} / n$.

Similarly, $M$ and $K$ are given by the action of the operators $\mathscr{M}$ and $\mathscr{K}$ on the covariance:

$$
\begin{aligned}
M & =\mathscr{M}\left(C_{0}+\delta C\right) \\
& \stackrel{\text { def }}{=} \frac{1}{4}\left[\left(\partial_{x_{a}} \partial_{x_{b}}-\partial_{y_{a}} \partial_{y_{b}}\right) \Delta_{a}^{-1} \Delta_{b}^{-1}\left(C_{0}+\delta C\right)\right]_{a=b}, \\
K & =\mathscr{K}\left(C_{0}+\delta C\right) \\
& \stackrel{\text { def }}{=} \frac{1}{4}\left[\left(\partial_{x_{a}} \partial_{x_{b}}+\partial_{y_{a}} \partial_{y_{b}}\right) \Delta_{a}^{-1} \Delta_{b}^{-1}\left(C_{0}+\delta C\right)\right]_{a=b} .
\end{aligned}
$$


For the jet perturbation $\bar{u}_{1}$ they are:

$$
\begin{aligned}
M & =M_{0}+\hat{M}_{1} \sin (n y)=\frac{\varepsilon}{8 \pi} \int d^{2} \mathbf{k}\left(k_{x}^{2}-k_{y}^{2}\right) \hat{Q}(\mathbf{k}) \\
& +\varepsilon \sin (n y) \int \frac{d^{2} \mathbf{k}}{4 \pi} \frac{i k_{x}\left(k_{x}^{2}-k_{y}^{2}-n k_{y}\right)\left(1-n^{2} / k^{2}\right)}{(\sigma+2) k^{2} k_{s}^{2}+2 i \beta n k_{x}\left(k_{y}+n / 2\right)} \hat{Q}(\mathbf{k}), \\
K & =K_{0}+\hat{K}_{1} \sin (i n y)=\frac{\varepsilon}{8 \pi} \int d^{2} \mathbf{k}\left(k_{x}^{2}+k_{y}^{2}\right) \hat{Q}(\mathbf{k}) \\
& +\varepsilon \sin (\text { iny }) \int \frac{d^{2} \mathbf{k}}{4 \pi} \frac{i k_{x}\left(k_{x}^{2}+k_{y}^{2}-n k_{y}\right)\left(1-n^{2} / k^{2}\right)}{(\sigma+2) k^{2} k_{s}^{2}+2 i \beta n k_{x}\left(k_{y}+n / 2\right)} \hat{Q}(\mathbf{k}) .
\end{aligned}
$$

To obtain the integrals we substitute $\hat{Q}$, move to polar coordinates as above and integrate over $k$ and $\phi$. The first integrals yield $M_{0}=\varepsilon \eta / 2$ and $K_{0}=\varepsilon / 2$. The second term in (A9) is a small correction that does not affect the jet acceleration and is therefore not calculated, while the second term in (A8) at the onset of instability is:

$$
\hat{M}_{1}=\int_{0}^{2 \pi} \frac{i N_{M}}{D_{f}+i \beta D_{\beta}} d \phi
$$

with $N_{M}=\varepsilon \cos \phi(\cos 2 \phi-n \sin \phi)\left(1-n^{2}\right)[1+\eta \cos (2 \phi)] / 4 \pi$. Therefore by numerically evaluating the integrals (A2) and (A10) for the most unstable jet with wavenumber $n=n_{c}$ and for the critical rate $\varepsilon=\varepsilon_{c}$ we calculate the stress tensor at the onset of instability.

We then estimate asymptotic values for the integrals and the stress tensor in the limits of both small and large values of the planetary vorticity gradient. In the limit $\beta \ll 1$ we expand the integrand in (A2) in powers of $\beta$ to obtain:

$$
\hat{f}_{1}=\sum_{j=1}^{\infty}(-i \beta)^{j} \int_{0}^{2 \pi} \frac{N_{f} D_{\beta}^{j-1}}{D_{f}^{j}} d \phi .
$$

The integrals of the odd powers of $\beta$ are zero, while the rest can be evaluated analytically through contour integration. For anisotropic forcing $(\eta=1)$ we obtain $\hat{f}_{1}=n^{2}\left(1-n^{2}\right) / 8+\mathscr{O}\left(\beta^{2}\right)$. The maximum value of $\hat{f}_{1}$ over the jet wavenumber $n$ is attained at $n_{c}=1 / \sqrt{2}$. The critical energy 
input rate is then according to (15) $\varepsilon_{c}=1 / \hat{f}_{1}=32$. For isotropic forcing $(\eta=0)$ we have:

$$
\hat{f}_{1}=\frac{\beta^{2} n^{4}}{1024}\left(48+\frac{5 \beta^{2} n^{2}\left(n^{4}+2 n^{2}-4\right)}{\left(1-n^{2}\right)^{2}}\right)+\mathscr{O}\left(\beta^{6}\right) .
$$

The maximum value of $\hat{f}_{1}$ can be evaluated approximately to occur for $n_{c}=1-\left(10 \beta^{2} / 768\right)^{1 / 3}+$ $\mathscr{O}\left(\beta^{4}\right)$, yielding a critical energy input rate $\varepsilon_{c} \simeq 64 / 3 \beta^{2}$. Therefore, from (A5) we calculate the momentum fluxes at the onset:

$$
\hat{N}_{1} \simeq 1+(\sqrt{2}-1) \eta
$$

Similarly for the eddy anisotropy, we expand the integrand in (A8) in powers of $\beta$. The non-zero leading order integral for $\eta=0$ is:

$$
\hat{M}_{1} \simeq \frac{\varepsilon \beta^{2} n^{3}\left(1-n^{2}\right)}{32 \pi} \int_{0}^{2 \pi} \frac{\cos ^{4} \phi(\cos 2 \phi-n \sin \phi)\left(8 \sin ^{3} \phi+12 n \sin ^{2} \phi+6 n^{2} \sin \phi+n^{3}\right)}{\left(1+2 n \sin \phi+n^{2}\right)^{4}} d \phi,
$$

which upon contour integration yields $\hat{M}_{1}=\varepsilon \beta^{3} n^{6} / 128\left(1-n^{2}\right)$. At the onset of instability $\left(\varepsilon=\varepsilon_{c}\right)$ and for the most unstable jet $\left(n=n_{c}\right)$ the eddy anisotropy is:

$$
\hat{M}_{1}=\left(\frac{12 \beta}{270}\right)^{1 / 3} .
$$

In the limit $\beta \gg 1$, the most unstable jet has small wavenumber (Srinivasan and Young 2012). We can therefore obtain an asymptotic expression for $n_{c}$ and $\varepsilon_{c}$ by expanding the integrand in (A2) in powers of $n$ but keeping $\beta n \sim \mathscr{O}(1)$ (since $\beta \gg 1$ ) to obtain:

$$
\begin{aligned}
\hat{f}_{1} & =\frac{n^{2}\left(1-n^{2}\right)}{4 \pi} \int_{0}^{2 \pi} \frac{\cos ^{2} \phi\left(1-4 \sin ^{2} \phi\right)(1+\eta \cos 2 \phi)}{(1+i \beta n \cos \phi \sin \phi)^{2}} d \phi \\
& +\mathscr{O}\left(n^{4}\right) .
\end{aligned}
$$

The integral can be evaluated analytically through contour integration yielding:

$$
\hat{f}_{1}=\frac{\left(1-n^{2}\right)\left\{-\left(16+6 \beta^{2} n^{2}\right)+\left(8+2 \beta^{2} n^{2}\right)\left[(1+\eta / 2) \sqrt{4+\beta^{2} n^{2}}-\eta\right]\right\}}{\beta^{2}\left(4+\beta^{2} n^{2}\right)^{3 / 2}} .
$$


The maximum value of $\hat{f}_{1}$ over the jet wavenumber $n$ is approximately attained at $n_{c} \simeq$ $[(3+\eta) /(2+\eta) \beta]^{1 / 3}$ and the critical energy input rate is $\varepsilon_{c} \simeq \beta^{2} /(2+\eta)$. The momentum fluxes are calculated from (A5):

$$
\hat{N}_{1}=\left(\frac{(2+\eta) \beta}{3+\eta}\right)^{1 / 3}
$$

To obtain $\hat{M}_{1}$ which is real, we rewrite (A10) in the form:

$$
\hat{M}_{1}=\frac{1}{\beta} \int_{0}^{2 \pi} \frac{i N_{M}}{\chi D_{f}+i D_{\beta}} d \phi
$$

where $\chi=1 / \beta$ and expand the integrand in powers of $\chi$. The leading order real term is:

$$
\hat{M}_{1} \simeq \frac{1}{\beta} \int_{0}^{2 \pi} \frac{N_{M} D_{\beta}}{\chi^{2} D_{f}^{2}+D_{\beta}^{2}} d \phi=\frac{1}{\beta} \int_{0}^{2 \pi} F_{\chi}(\phi, n) d \phi .
$$

For the angles $\phi$ for which $D_{\beta} \sim \mathscr{O}(1)$, the integrand is order one. However, if $D_{\beta} \sim \mathscr{O}\left(\beta^{-1}\right)$ for some angle $\phi_{r}$, then the denominator is order $\chi$ and the integrand is large. The angles $\phi_{r}$ are the roots of $D_{\beta}$ which are at $\phi_{1}=\pi / 2, \phi_{3}=3 \pi / 2$ and the two angles satisfying $\phi_{2,4}=-\sin ^{-1}(n / 2)$.

To calculate asymptotic approximations to the integral $\hat{M}_{1}$, we split the range of integration to a small range close to the roots of $D_{\beta}$ for which the integrand is large, $I^{(\mathrm{R})}$, and to a range away from the roots of $D_{\beta}, I^{(\mathrm{NR})}$ :

$$
\hat{M}_{1}=\sum_{j=1}^{4}[\underbrace{\int_{\phi_{j-1}+\delta \phi}^{\phi_{j}-\delta \phi} F_{\chi}(\phi, n) d \phi}_{I_{j}^{(\mathrm{NR})}}+\underbrace{\int_{\phi_{j}-\delta \phi}^{\phi_{j}+\delta \phi} F_{\chi}(\phi, n) d \phi}_{I_{j}^{\mathrm{R})}}],
$$

where the range $\delta \phi=a \chi$, with $a$ an order one parameter. Asymptotic approximations to the integral over the two ranges are then found separately (cf. Hinch (1991)).

To calculate $I^{(\mathrm{R})}$, we rescale the angle close to the roots $\phi=\phi_{j}+a \chi u$, to obtain:

$$
I_{j}^{(\mathrm{R})}=\chi \int_{-a}^{a} \frac{N_{M}\left(\phi_{j}+a \chi u\right) D_{\beta}\left(\phi_{j}+a \chi u\right)}{D_{f}^{2}\left(\phi_{j}+a \chi u\right)+\chi^{2} D_{\beta}^{2}\left(\phi_{j}+a \chi u\right)} d u+\mathscr{O}\left(\chi^{-3}\right) .
$$


We then Taylor expand the integrand in powers of $\chi$ :

$$
\begin{aligned}
& I_{j}^{(\mathrm{R})}=\int_{-a}^{a} \frac{N_{M, j} D_{\beta, j}^{\prime} u}{D_{f, j}^{2}+D_{\beta, j}^{\prime}{ }^{2} u^{2}} d u \\
+ & \chi \int_{-a}^{a} \frac{\left[D_{f, j}^{2}\left(2 D_{\beta, j}^{\prime} N_{M, j}^{\prime}+N_{M, j} D_{\beta, j}^{\prime \prime}\right)-4 D_{f, j} D_{f, j}^{\prime} D_{\beta, j}^{\prime} N_{M, j}\right] u^{2}}{2\left(D_{f, j}^{2}+D_{\beta, j}^{\prime}{ }^{2}\right)^{2}} d u \\
+ & \chi \int_{-a}^{a} \frac{D_{\beta, j}^{\prime}{ }^{2}\left(2 D_{\beta, j}^{\prime} N_{M, j}^{\prime}-D_{\beta, j}^{\prime \prime} N_{M, j}\right) u^{4}}{2\left(D_{f, j}^{2}+D_{\beta, j}^{\prime}{ }^{2} u^{2}\right)^{2}} d u+\mathscr{O}\left(\chi^{2}\right),
\end{aligned}
$$

where primed quantities are derivatives with respect to angle and the subscript $j$ denotes the value of the relative function at $\phi_{j}$. Evaluation of the integrals and summation over all roots yields:

$$
I^{(\mathrm{R})}=\frac{\varepsilon a \chi\left(1-n^{2}\right)^{2}}{4 \pi\left(1-n^{2} / 4\right)}+\frac{\varepsilon \chi|n|\left(1-n^{2}\right)\left(n^{2}-2\right)}{8\left(1-n^{2} / 4\right)^{2}} .
$$

To calculate $I^{(\mathrm{NR})}$, we expand the integrand for $\chi \ll 1$, to obtain to leading order:

$$
I^{(\mathrm{NR})}=\sum_{j=1}^{4} \int_{\phi_{j-1}+\delta \phi}^{\phi_{j}-\delta \phi} \frac{N_{M}}{D_{\beta}} d \phi+\mathscr{O}\left(\chi^{2}\right) .
$$

To evaluate the sum of the integrals, we move into the complex plane by setting $z=e^{i \phi}$. The path of integration $\mathscr{C}_{0}$ that lies on the unit circle is shown by the thick line in Fig. 9 and excludes the region of width $2 \delta \phi$ close to the angles $\phi_{j}$. To evaluate the integrals, we add the small paths $\mathscr{C}_{j}$, with $j=$ $1, \ldots, 4$ around the angles $\phi_{j}$ that are also shown in Fig. 9, to obtain a closed path $\Sigma \mathscr{C}$. The paths $\mathscr{C}_{j}$ are defined by $z=e^{i \phi_{j}}+R e^{i \vartheta}$, where $R=\sin \delta \phi / \sin (\pi+\omega)$, and $\omega=\cot ^{-1}[(\cos \delta \phi-1) / \sin \delta \phi]$. The angle $\vartheta$ is limited by $\left|\vartheta-\phi_{j}-\pi\right| \leq \omega$. The integrand has poles at the angles $\phi_{j}$ that are outside the closed path and at $z=0$, so by Cauchy's theorem the integral over the closed path $\Sigma \mathscr{C}$ is:

$$
\int_{\Sigma \mathscr{C}} \frac{N_{M}}{i z D_{\beta}} d z=2 \pi \operatorname{Res}(0) \text {. }
$$

The residue is zero yielding:

$$
I^{(\mathrm{NR})}=\int_{\mathscr{C}_{0}} \frac{N_{M}}{i z D_{\beta}} d z=-\sum_{j=1}^{4} \int_{\mathscr{C}_{j}} \frac{N_{M}}{i z D_{\beta}} d z .
$$


Evaluating the integrands at the paths $\mathscr{C}_{j}$, expanding the functions in powers of $\delta \phi$ and calculating the resulting integrals yields:

$$
I^{(\mathrm{NR})}=-\frac{\varepsilon \delta \phi\left(1-n^{2}\right)^{2}}{4 \pi\left(1-n^{2} / 4\right)} .
$$

Adding (A24) and (A28) yields $\hat{M}_{1}=\varepsilon|n|\left(1-n^{2}\right)\left(n^{2}-2\right) / 8 \beta^{2}\left(1-n^{2} / 4\right)^{2}$. At the onset of zonostrophic instability $n=n_{c}$ and:

$$
\hat{M}_{1} \simeq\left(\frac{3}{2^{10} \beta}\right)^{1 / 3}
$$

\section{APPENDIX B}

\section{Calculation of the eddy variance ellipse during the equilibration of zonostrophic instability}

In this Appendix we study the equilibration of zonostrophic instability and calculate how the variance ellipse changes during the development of the instability. To obtain the weakly non-linear dynamics governing the jet evolution near the onset of zonostrophic instability, we assume that the energy input rate is slightly supercritical $\varepsilon=\varepsilon_{c}\left(1+\mu^{2}\right)$, where $\mu \ll 1$. Following the discussion in section 5 we assume that the most unstable jet with wavenumber $n_{c}$ evolves on a slow time scale $T=\mu^{2} t: \bar{u}_{1}=A(T) e^{i n_{c} y}+A^{*}(T) e^{-i n_{c} y}$. We expand the velocity and the covariance as a series in $\mu$ (Eqs. (29)-(30)), substitute (29)-(30) in (8)-(9) and collect terms with equal powers of $\mu$. Details on the calculation can be found in Bakas et al. (2019). Here, we will only recite the main results.

As described in section 5, the order one terms yield the homogeneous equilibrium with $C_{0}=$ $\varepsilon_{c} Q / 2$, while the order $\mu$ terms yield the eigenfunction (31). At order $\mu^{2}$, the quasi-linear interaction of the infinitesimal jet $\bar{u}_{1}$ with the perturbation in the eddy covariance $C_{1}$ generates a covariance correction with a homogeneous component and a component at the double harmonic $2 n_{c}$

that is given by (32). The homogeneous component $\hat{C}_{2}^{0}\left(\mathbf{x}_{a}-\mathbf{x}_{b}, T\right)=\varepsilon_{c} Q / 2+\hat{C}_{2}^{0, N L}\left(\mathbf{x}_{a}-\mathbf{x}_{b}, T\right)$ contains two parts. The first part is the contribution of the homogeneous covariance $C_{0}=$ 
$\left(1+\mu^{2}\right) \varepsilon_{c} Q / 2$ at order $\mu^{2}$. The second part

$$
\begin{aligned}
& \hat{C}_{2}^{0, N L}\left(\mathbf{x}_{a}-\mathbf{x}_{b}, T\right)=\mathscr{L}^{-1} \mathscr{J}_{A e^{i n_{c} y}}\left(\hat{C}_{1}^{1}\right)^{*} e^{-i n_{c}\left(y_{a}+y_{b}\right) / 2} \\
+ & \mathscr{L}^{-1} \mathscr{J}_{A^{*}} e^{-i n_{c} y} \hat{C}_{1}^{1} e^{i n_{c}\left(y_{a}+y_{b}\right) / 2},
\end{aligned}
$$

is due to the quasi-linear interaction of $\bar{u}_{1}$ with $C_{1}$. In (B1), the operators $\mathscr{L}=$ $-\beta\left(\partial_{x_{a}} \Delta_{a}^{-1}+\partial_{x_{b}} \Delta_{b}^{-1}\right)-2$ and $\mathscr{J}_{\bar{u}}=-\bar{u}_{a} \partial_{x_{a}}-\bar{u}_{b} \partial_{x_{b}}+\partial_{y_{a y} y_{a}}^{2} \bar{u}_{a} \partial_{x_{a}} \Delta_{a}^{-1}+\partial_{y_{b} y_{b}}^{2} \bar{u}_{b} \partial_{x_{b}} \Delta_{b}^{-1}$ are the linear and non-linear parts of the operator $\mathscr{A}_{a}+\mathscr{A}_{b}$. The double harmonic component drives a jet with velocity $\bar{u}_{2}=\alpha_{2} A^{2}(T) e^{2 i n c y}+$ c.c. and is given by

$$
\begin{aligned}
\hat{C}_{2}^{2} & =e^{-2 i n_{c}\left(y_{a}+y_{b}\right) / 2} \mathscr{L}^{-1} \mathscr{J}_{A e^{i n_{c} y}} \hat{C}_{1}^{1} e^{i n_{c}\left(y_{a}+y_{b}\right) / 2} \\
& +e^{-2 i n_{c}\left(y_{a}+y_{b}\right) / 2} \mathscr{L}^{-1} \mathscr{J}_{\alpha_{2} A^{2} e^{2 i n_{c} y}}\left(\varepsilon_{c} Q / 2\right)
\end{aligned}
$$

The constant $\alpha_{2}$ is an order one parameter given by:

$$
\alpha_{2}=\frac{\frac{\varepsilon_{c}}{2} \int \frac{d^{2} \mathbf{k}}{2 \pi} \frac{i n_{c} k_{x}^{3}\left(k^{2}-n_{c}^{2}\right)}{k^{2} k_{2}^{2}+i \beta n_{c} k_{x} k_{y, 1}}\left\{\frac{k_{y, 2}\left(k_{2}^{2}-n_{c}^{2}\right)}{k^{2} k_{4}^{2}+2 i \beta n_{c} k_{x} k_{y, 2}}-\frac{k_{y} k_{2}^{2}\left(k^{2}-n_{c}^{2}\right)}{k^{2}\left(k_{-2}^{2} k_{2}^{2}+2 i \beta n_{c} k_{x} k_{y}\right)}\right\} \hat{Q}(\mathbf{k})}{\varepsilon_{c} \int \frac{d^{2} \mathbf{k}}{2 \pi} \frac{n_{c} k_{x}^{2} k_{y, 2}\left(k^{2}-4 n_{c}^{2}\right)}{k^{2}\left(k^{2} k_{4}^{2}+2 i \beta n_{c} k_{x} k_{y, 2}\right)} \hat{Q}(\mathbf{k})-1},
$$

where $k_{y, j} \stackrel{\text { def }}{=} k_{y}+j n_{c} / 2$ and $k_{j}^{2} \stackrel{\text { def }}{=} k_{x}^{2}+k_{y, j}^{2}$ for any integer $j$ (eg. Equation (B7) of Bakas et al. (2019)).

At order $\mu^{3}$, the quasi-linear interaction between the double harmonic jet $\bar{u}_{2}$ and the eddy covariance $C_{1}$ as well as the interaction between $\bar{u}_{1}$ and $C_{2}$ generate the covariance correction

$$
C_{3}=\hat{C}_{3}^{1} e^{i n_{c}\left(y_{a}+y_{b}\right) / 2}+\hat{C}_{3}^{3} e^{3 i n_{c}\left(y_{a}+y_{b}\right) / 2}+\text { c.c.. }
$$

The $3 n_{c}$ component drives a jet with velocity $\bar{u}_{3}=\alpha_{3}|A|^{2} A e^{3 i n_{c} y}+$ c.c and is given by

$$
\begin{aligned}
\hat{C}_{3}^{3} & =e^{-3 i n_{c}\left(y_{a}+y_{b}\right) / 2} \mathscr{L}^{-1} \mathscr{J}_{A e^{i n_{c} y}} \hat{C}_{2}^{2} e^{2 i n_{c}\left(y_{a}+y_{b}\right) / 2} \\
& +e^{-3 i n_{c}\left(y_{a}+y_{b}\right) / 2} \mathscr{L}^{-1} \mathscr{J}_{\alpha_{3}|A|^{2} A e^{3 i n_{c} y}\left(\varepsilon_{c} Q / 2\right) .}
\end{aligned}
$$


The constant $\alpha_{3}$ is an order one parameter. The component

$$
\hat{C}_{3}^{1}\left(\mathbf{x}_{a}-\mathbf{x}_{b}, T\right)=\hat{C}_{1}^{1, h}\left(\mathbf{x}_{a}-\mathbf{x}_{b}, T\right)+\underbrace{\hat{C}_{3}^{1, e c}\left(\mathbf{x}_{a}-\mathbf{x}_{b}, T\right)+\hat{C}_{3}^{1,12}\left(\mathbf{x}_{a}-\mathbf{x}_{b}, T\right)}_{\hat{C}_{3}^{1, N L}},
$$

contains three parts. The first part

$$
\hat{C}_{3}^{1, h}=e^{-i n_{c}\left(y_{a}+y_{b}\right) / 2} \mathscr{L}^{-1} \mathscr{J}_{A e^{i n_{c} y}}\left(\varepsilon_{c} Q / 2\right),
$$

is due to the interaction of $\bar{u}_{1}$ with the homogeneous covariance. The second part is

$$
\hat{C}_{3}^{1, e c}=e^{-i n_{c}\left(y_{a}+y_{b}\right) / 2} \mathscr{L}^{-1} \mathscr{J}_{A e^{i n_{c}}} \hat{C}_{0}^{2, N L},
$$

is due to the quasi-linear interactions of $\bar{u}_{1}$ with the homogeneous correction $\hat{C}_{2}^{0}$ and is referred in Bakas et al. (2019) as the energy correction term. The third part

$$
\begin{aligned}
\hat{C}_{3}^{1,12} & =e^{-i n_{c}\left(y_{a}+y_{b}\right) / 2} \mathscr{L}^{-1} \mathscr{J}_{\alpha_{2} A^{2} e^{2 i n_{c} y}}\left(\hat{C}_{1}^{1}\right)^{*} e^{-i n_{c}\left(y_{a}+y_{b}\right) / 2} \\
& +e^{-i n_{c}\left(y_{a}+y_{b}\right) / 2} \mathscr{L}^{-1} \mathscr{J}_{A^{*} e^{-i n_{c} y}} \hat{C}_{2}^{2} e^{2 i n_{c}\left(y_{a}+y_{b}\right) / 2},
\end{aligned}
$$

is due to the quasi-linear interactions of $\bar{u}_{1}$ with $\hat{C}_{2}^{2} e^{2 i n_{c}\left(y_{a}+y_{b}\right) / 2}$ and $\bar{u}_{2}$ with $C_{1}$.

The $\hat{C}_{3}^{1}$ component produces a $\mu^{3}$ order correction to the vorticity fluxes at wavenumber $n_{c}$ and it also produces secular terms that vanish only if the amplitude $A$ satisfies the Ginzburg-Landau (G-L) equation

$$
c_{1} \partial_{T} A=A-c_{3}|A|^{2} A
$$

where

$$
\begin{gathered}
c_{1}=1+\frac{\varepsilon_{c}}{4} \int \frac{d^{2} \mathbf{k}}{2 \pi} \frac{n_{c} k_{x}^{2} k_{y, 1} k_{2}^{2}\left(k^{2}-n_{c}^{2}\right)}{\left(k^{2} k_{2}^{2}+i \beta n_{c} k_{x} k_{y, 1}\right)^{2}} \hat{Q}(\mathbf{k}) \\
c_{3}=\frac{\varepsilon_{c}}{4} \int \frac{d^{2} \mathbf{k}}{2 \pi} \frac{n_{c} k_{x}^{4} k_{2}^{2}\left(k_{2}^{2}-n_{c}^{2}\right)\left(k^{2}-n_{c}^{2}\right)^{2}}{\left|k^{2} k_{2}^{2}+i \beta n_{c} k_{x} k_{y, 1}\right|^{2}} \times \\
{\left[\frac{2 k_{y, 1}}{k^{2} k_{2}^{2}+i \beta n_{c} k_{x} k_{y, 1}}-\frac{k_{y,-1}}{k^{2} k_{-2}^{2}+i \beta n_{c} k_{x} k_{y,-1}}-\frac{k_{y, 3}}{k_{2}^{2} k_{4}^{2}+i \beta n_{c} k_{x} k_{y, 3}}\right] \hat{Q}(\mathbf{k}) .}
\end{gathered}
$$


(Eqs. (B13) and (B16) of Bakas et al. (2019)). The linear term in the (G-L) equation (B10) is due to $\hat{C}_{3}^{1, h}$ and drives the zonostrophic instability. The non-linear term is due to $\hat{C}_{3}^{1, N L}$ and equilibrates the instability.

To obtain the eddy variance ellipse during the instability evolution as well as for the equilibrated jet, we calculate from the covariance (30) the momentum fluxes, the eddy anisotropy and the eddy kinetic energy for a sinusoidal mean flow $\bar{u}_{1}=A(T) \sin \left(n_{c} y\right)$ as:

$$
\begin{gathered}
N=\mu N_{1}+\mu^{2} N_{2}+\mu^{3} N_{3}+\cdots \\
=\mu \operatorname{Im}\left(\hat{N}_{1}^{1} e^{i n_{c} y}\right)+\mu^{2} \operatorname{Im}\left(\hat{N}_{2}^{2} e^{2 i n_{c} y}\right) \\
+\mu^{3} \operatorname{Im}\left(\hat{N}_{3}^{1} e^{i n_{c} y}+\hat{N}_{3}^{3} e^{3 i n_{c} y}\right)+\cdots, \\
M=M_{0}+\mu M_{1}+\mu^{2} M_{2}+\mu^{3} M_{3}+\cdots \\
=M_{0}+\mu \operatorname{Im}\left(\hat{M}_{1}^{1} e^{i n_{c} y}\right)+\mu^{2} \operatorname{Im}\left(\hat{M}_{2}^{0}+\hat{M}_{2}^{2} e^{2 i n_{c} y}\right) \\
+\mu^{3} \operatorname{Im}\left(\hat{M}_{3}^{1} e^{i n_{c} y}+\hat{M}_{3}^{3} e^{3 i n_{c} y}\right)+\cdots \\
K=K_{0}+\mu K_{1}+\mu^{2} K_{2}+\cdots=K_{0}+\mu \operatorname{Im}\left(\hat{K}_{1}^{1} e^{i n_{c} y}\right) \\
+\mu^{2} \operatorname{Im}\left(\hat{K}_{2}^{0}+\hat{K}_{2}^{2} e^{2 i n_{c} y}\right)+\cdots \\
K
\end{gathered}
$$

with $\quad \hat{N}_{j}^{l}=e^{-i l n_{c} y} \mathscr{N}\left(\hat{C}_{j}^{l} e^{i l n_{c}\left(y_{a}+y_{b}\right) / 2}\right), \quad \hat{M}_{j}^{l}=e^{-i l n_{c} y} \mathscr{M}\left(\hat{C}_{j}^{l} e^{i l n_{c}\left(y_{a}+y_{b}\right) / 2}\right), \quad \hat{K}_{j}^{l}=$ $e^{-i l n_{c} y} \mathscr{K}\left(\hat{C}_{j}^{l} e^{i l n_{c}\left(y_{a}+y_{b}\right) / 2}\right), M_{0}=\varepsilon_{c} \eta / 2$ and $K_{0}=\varepsilon_{c} / 2$.

For isotropic forcing $(\eta=0)$, the tilt angle is

$$
\theta=\theta_{1}+\mu \theta_{2}+\mu^{2} \theta_{3}+\cdots
$$


where $\theta_{1}=-(1 / 2) \arctan \left(N_{1} / M_{1}\right), \theta_{2}=-\left(M_{1} N_{2}-M_{2} N_{1}\right) / 2\left(N_{1}^{2}+M_{1}^{2}\right)$ and the third order correction

$$
\begin{aligned}
\theta_{3} & =\theta_{3,2}+\theta_{3,3} \\
& =-\frac{\left(M_{1} M_{2}+N_{1} N_{2}\right)\left(M_{2} N_{1}-M_{1} N_{2}\right)}{2\left(N_{1}^{2}+M_{1}^{2}\right)^{2}} \\
& -\frac{M_{1} N_{3}-M_{3} N_{1}}{2\left(N_{1}^{2}+M_{1}^{2}\right)}
\end{aligned}
$$

contains two terms. The first $\left(\theta_{3,2}\right)$ involves the second-order quantities $N_{2}, M_{2}$ and the second $\left(\theta_{3,3}\right)$ involves the third order quantities $N_{3}, M_{3}$. Similarly, the anisotropy parameter is given by:

$$
\gamma=\mu \gamma_{1}+\mu^{2} \gamma_{2}+\mu^{3} \gamma_{3}+\cdots
$$

where

$$
\gamma_{1}=\frac{\sqrt{N_{1}^{2}+M_{1}^{2}}}{K_{0}}, \gamma_{2}=\frac{K_{0}\left(M_{1} M_{2}+N_{1} N_{2}\right)-K_{1}\left(N_{1}^{2}+M_{1}^{2}\right)}{K_{0}^{2} \sqrt{N_{1}^{2}+M_{1}^{2}}},
$$

and the third order correction $\gamma_{3}=\gamma_{3,2}+\gamma_{3,3}$ is the sum of the terms

$$
\begin{gathered}
\gamma_{3,2}=\frac{2\left(K_{1}^{2}-K_{0} K_{2}\right)\left(N_{1}^{2}+M_{1}^{2}\right)^{2}-2 K_{0} K_{1}\left(M_{1} M_{2}+N_{1} N_{2}\right)\left(N_{1}^{2}+M_{1}^{2}\right)+K_{0}^{2}\left(M_{2} N_{1}-N_{2} M_{1}\right)^{2}}{2 K_{0}^{3}\left(N_{1}^{2}+M_{1}^{2}\right)^{3 / 2}} \\
\gamma_{3,3}=\frac{N_{1} N_{3}+M_{1} M_{3}}{K_{0} \sqrt{N_{1}^{2}+M_{1}^{2}}}
\end{gathered}
$$

that contain second and third order quantities respectively.

The vorticity fluxes forcing the mean flow are:

$$
\overline{v^{\prime} \zeta^{\prime}}=\mu f_{1}+\mu^{2} f_{2}+\mu^{3} f_{3}
$$

where

$$
f_{1}=-K_{0} \gamma_{1}^{\prime} \sin 2 \theta_{1}-2 K_{0} \gamma_{1} \theta_{1}^{\prime} \cos 2 \theta_{1},
$$

are the fluxes at leading order that exactly overcome dissipation at the onset of instability and primed quantities denote differentiation with respect to latitude. At the next order, the fluxes $f_{2}$ 
drive the mean flow $\bar{u}_{2}$ with wavenumber $2 n_{c}$. At the third order,

$$
\begin{aligned}
f_{3} & =-K_{0}\left(\gamma_{3,3}^{\prime}-4 \theta_{1}^{\prime} \gamma_{1} \theta_{3,3}\right) \sin 2 \theta_{1} \\
& -2 K_{0}\left(\theta_{3,3}^{\prime} \gamma_{1}+\theta_{1}^{\prime} \gamma_{3,3}+\gamma_{1}^{\prime} \theta_{3,3}\right) \cos 2 \theta_{1},
\end{aligned}
$$

as the sum of all terms that involve second-order quantities is exactly zero. Therefore $f_{3}$ is a function of the third order momentum fluxes $N_{3}$ and eddy anisotropy $M_{3}$. We observe that $\left|\hat{N}_{3}^{3}\right| \ll$ $\left|\hat{N}_{3}^{1}\right|$ and $\left|\hat{M}_{3}^{3}\right| \ll\left|\hat{M}_{3}^{1}\right|$. As a result:

$$
N_{3} \simeq \operatorname{Im}\left(\hat{N}_{3}^{1} e^{i n_{c} y}\right)=\mathscr{N}\left(\hat{C}_{3}^{1, h}+\hat{C}_{3}^{1, N L}\right)=N_{1}+N_{3}^{N L}
$$

where $N_{3}^{N L}=N_{3}^{e c}+N_{3}^{12}=\mathscr{N}\left(\hat{C}_{3}^{1, e c}\right)+\mathscr{N}\left(\hat{C}_{3}^{1,12}\right)$. Similarly $M_{3} \simeq M_{1}+M_{3}^{N L}$ with $M_{3}^{N L}=$ $M_{3}^{e c}+M_{3}^{12}=\mathscr{M}\left(\hat{C}_{3}^{1, e c}\right)+\mathscr{M}\left(\hat{C}_{3}^{1,12}\right)$. Substituting in (B17) and (B21) we obtain:

$$
\begin{aligned}
\theta_{3,3} & =\theta_{3, N L}=\theta_{3, e c}+\theta_{3,12} \\
& =-\frac{M_{1} N_{3}^{e c}-M_{3}^{e c} N_{1}}{2\left(N_{1}^{2}+M_{1}^{2}\right)}-\frac{M_{1} N_{3}^{12}-M_{3}^{12} N_{1}}{2\left(N_{1}^{2}+M_{1}^{2}\right)}, \\
\gamma_{3,3} & =\gamma_{1}+\gamma_{3, N L}=\gamma_{1}+\left(\gamma_{3, e c}+\gamma_{3,12}\right) \\
& =\gamma_{1}+\frac{N_{1} N_{3}^{e c}+M_{1} M_{3}^{e c}}{K_{0} \sqrt{N_{1}^{2}+M_{1}^{2}}}+\frac{N_{1} N_{3}^{12}+M_{1} M_{3}^{12}}{K_{0} \sqrt{N_{1}^{2}+M_{1}^{2}}},
\end{aligned}
$$

where the subscripts (or superscripts) $e c$ and 12 denote the contribution of the energy correction term $\left(\hat{C}_{3}^{1, e c}\right)$ and the term due to the interaction of the single harmonic jet-eddy components with their double harmonic counterparts $\left(\hat{C}_{3}^{1,12}\right)$ to $\theta_{3, N L}$ and $\gamma_{3, N L}$.

Therefore the vorticity fluxes at third order are:

$$
\begin{aligned}
f_{3} & =f_{1}-K_{0}\left(\gamma_{3, N L}^{\prime}-4 \theta_{1}^{\prime} \gamma_{1} \theta_{3, N L}\right) \sin 2 \theta_{1} \\
& -2 K_{0}\left(\theta_{3, N L}^{\prime} \gamma_{1}+\theta_{1}^{\prime} \gamma_{3, N L}+\gamma_{1}^{\prime} \theta_{3, N L}\right) \cos 2 \theta_{1} .
\end{aligned}
$$

The first term corresponds to the linear term in (B10) and drives the instability, while the other two terms correspond to the non-linear term in (B10) that equilibrate the instability. For small jet 
amplitude $A$, these terms are $|A|^{2}$ smaller than the first term and (B28) is the leading order of

$$
\begin{aligned}
f_{3} & \simeq-K_{0}\left(\gamma_{1}+\gamma_{3, N L}\right)^{\prime} \sin \left(2 \theta_{1}+2 \theta_{3, N L}\right) \\
& -2 K_{0}\left(\gamma_{1}+\gamma_{3, N L}\right) \cos \left(2 \theta_{1}+2 \theta_{3, N L}\right) .
\end{aligned}
$$

Therefore $\theta_{3, N L}$ and $\gamma_{3, N L}$ are the non-linear corrections to the tilt angle and the eddy anisotropy parameter respectively.

For anisotropic forcing $(\eta=1)$, the tilt angle and the anisotropy parameter are given by:

$$
\theta=\mu \theta_{1}+\mu^{2} \theta_{2}+\mu^{3} \theta_{3}+\cdots, \quad \gamma=\gamma_{0}+\mu \gamma_{1}+\mu^{2} \gamma_{2}+\cdots,
$$

where $\gamma_{0}=M_{0} / K_{0}$ is the anisotropy parameter for the homogeneous equilibrium, the corrections

$$
\begin{aligned}
\theta_{1}=-N_{1} / 2 M_{0}, \theta_{2}=\left(M_{1} N_{1}\right. & \left.-M_{0} N_{2}\right) / 2 M_{0}^{2}, \\
\gamma_{1} & =\frac{K_{0} M_{1}-K_{1} M_{0}}{K_{0}^{2}}, \\
\gamma_{2} & =\frac{2 M_{0}^{2}\left(K_{1}^{2}-K_{0} K_{2}\right)+K_{0}^{2}\left(2 M_{0} M_{2}+N_{1}^{2}\right)}{} \\
& -\frac{2 K_{1} M_{1}}{2 K_{0}^{2} M_{0}} .
\end{aligned}
$$

contain second-order quantities and the third order correction

$$
\begin{aligned}
\theta_{3} & =\theta_{3,2}+\theta_{3,3} \\
& =\frac{N_{1}\left(N_{1}^{2}-3 M_{1}^{2}\right)+3 M_{0}\left(M_{2} N_{1}+M_{1} N_{2}\right)}{6 M_{0}^{3}} \\
& -\frac{N_{3}}{2 M_{0}},
\end{aligned}
$$

has two terms containing second-order quantities $\left(\theta_{3,2}\right)$ and third order quantities $\left(\theta_{3,3}\right)$. The vorticity fluxes are given by (B22), where

$$
f_{1}=2 \gamma_{0} K_{0} \theta_{1}^{\prime},
$$

balances the mean flow dissipation and $f_{2}$ drives $\bar{u}_{2}$. The third order correction is

$$
f_{3}=2 \gamma_{0} K_{0} \theta_{3,3}^{\prime},
$$


as again the terms involving second-order quantities cancel out. Substituting (B25) in (B32) we obtain $\theta_{3,3}=\theta_{1}+\theta_{3, N L}$, with

$$
\theta_{3, N L}=\theta_{3, e c}+\theta_{3,12}=-\frac{N_{3}^{e c}}{2 M_{0}}-\frac{N_{3}^{12}}{2 M_{0}} .
$$

Therefore $\theta_{3, N L}$ is the non-linear correction to the tilt angle that brings about the equilibration of the flow according to (B34). We found numerically that $N_{3}^{N L}=-|A|^{2} N_{1}$ and as a result $\theta_{3, N L}=$ $|A|^{2}\left(\pi / 2-\theta_{1}\right)$.

\section{References}

Bakas, N. A., N. C. Constantinou, and P. J. Ioannou, 2015: S3T stability of the homogeneous state of barotropic beta-plane turbulence. J. Atmos. Sci., 72 (5), 1689-1712, doi: 10.1175/JAS-D-14-0213.1.

Bakas, N. A., N. C. Constantinou, and P. J. Ioannou, 2019: Statistical state dynamics of weak jets in barotropic beta-plane turbulence. J. Atmos. Sci., 76, 919-945, doi:10.1175/JAS-D-18-0148.1.

Bakas, N. A., and P. J. Ioannou, 2013a: Emergence of large scale structure in barotropic $\beta$-plane turbulence. Phys. Rev. Lett., 110, 224 501, doi:10.1103/PhysRevLett.110.224501.

Bakas, N. A., and P. J. Ioannou, 2013b: On the mechanism underlying the spontaneous emergence of barotropic zonal jets. J. Atmos. Sci., 70 (7), 2251-2271, doi:10.1175/JAS-D-12-0102.1.

Bakas, N. A., and P. J. Ioannou, 2014: A theory for the emergence of coherent structures in betaplane turbulence. J. Fluid Mech., 740, 312-341, doi:10.1017/jfm.2013.663.

Cessi, P., and M. Fantini, 2004: The eddy-driven Thermocline. J. Phys. Oceanogr., 34, 2642-2658. 
Constantinou, N. C., B. F. Farrell, and P. J. Ioannou, 2014a: Emergence and equilibration of jets in beta-plane turbulence: applications of Stochastic Structural Stability Theory. J. Atmos. Sci., 71 (5), 1818-1842, doi:10.1175/JAS-D-13-076.1.

Constantinou, N. C., A. Lozano-Durán, M.-A. Nikolaidis, B. F. Farrell, P. J. Ioannou, and J. Jiménez, 2014b: Turbulence in the highly restricted dynamics of a closure at second order: comparison with DNS. J. Phys. Conf. Ser., 506, 012 004, doi:10.1088/1742-6596/506/1/012004.

Constantinou, N. C., and J. B. Parker, 2018: Magnetic suppression of zonal flows on a beta plane. Astrophys. J., 863 (1), 46, doi:10.3847/1538-4357/aace53.

Danabasoglu, G., J. C. McWilliams, and P. R. Gent, 1994: The role of mesoscale eddy transports in the global ocean circulation. Science, 264, 1123-1126.

DelSole, T., 2004: Stochastic models of quasigeostrophic turbulence. Surv. Geophys., 25, 107149, doi:10.1023/B:GEOP.0000028160.75549.0d.

Eady, E. A., 1949: Long waves and cyclone waves. Tellus, 1 (3), 33-52, doi:10.3402/tellusa.v1i3. 8507.

Farrell, B. F., and P. J. Ioannou, 2003: Structural stability of turbulent jets. J. Atmos. Sci., 60, 2101-2118, doi:10.1175/1520-0469(2003)060〈2101:SSOTJ $\rangle 2.0 . C O ; 2$.

Farrell, B. F., and P. J. Ioannou, 2007: Structure and spacing of jets in barotropic turbulence. $J$. Atmos. Sci., 64, 3652-3665, doi:10.1175/JAS4016.1.

Farrell, B. F., and P. J. Ioannou, 2008: Formation of jets by baroclinic turbulence. J. Atmos. Sci., 65, 3353-3375, doi:10.1175/2008JAS2611.1. 
Farrell, B. F., and P. J. Ioannou, 2009a: Emergence of jets from turbulence in the shallowwater equations on an equatorial beta plane. J. Atmos. Sci., 66, 3197-3207, doi:10.1175/ 2009JAS2941.1.

Farrell, B. F., and P. J. Ioannou, 2009b: A stochastic structural stability theory model of the drift wave-zonal flow system. Phys. Plasmas, 16, 112 903, doi:10.1063/1.3258666.

Farrell, B. F., P. J. Ioannou, and M. A. Nikolaidis, 2017: Instability of the roll-streak structure induced by background turbulence in pretransitional Couette flow. Phys. Rev. Fluids, 2 (3), 034 607, doi:10.1103/PhysRevFluids.2.034607.

Fitzgerald, J. G., and B. F. Farrell, 2018a: Statistical State Dynamics of vertically sheared horizontal flows in two-dimensional stratified turbulence. J. Fluid Mech., 854, 544-590, doi: 10.1017/jfm.2018.560.

Fitzgerald, J. G., and B. F. Farrell, 2018b: Vertically sheared horizontal flow-forming instability in stratified turbulence: Analytical linear stability analysis of Statistical State Dynamics equilibria. J. Atmos. Sci., 75, 4201-4227, doi:10.1175/JAS-D-18-0075.1.

Galperin, B. H., S. Sukoriansky, and N. Dikovskaya, 2010: Geophysical flows with anisotropic turbulence and dispersive waves: flows with a $\beta$-effect. Ocean Dyn., 60, 427-441, doi:10.1007/ s10236-010-0278-2.

Hinch, E. J., 1991: Perturbation Methods. Cambridge University Press.

Hopf, E., 1952: Statistical hydromechanics and functional calculus. J. Ration. Mech. Anal., 1, 87-123, doi:10.1512/iumj.1952.1.51004.

Hoskins, B., I. N. James, and G. H. White, 1983: The shape, propagation, and mean flow interaction of large-scale weather systems. J. Atmos. Sci., 40, 1595-1612. 
Lilly, D. K., 1969: Numerical simulation of two-dimensional turbulence. Phys. Fluids, 12, II240II249, doi:10.1063/1.1692444.

Mak, J., J. R. Maddison, D. P. Marshall, and D. R. Munday, 2018: Implementation of a geometrically informed and energetically constrained mesoscale eddy parameterization in an ocean circulation model. J. Phys. Oceanogr., 48, 2363-2382.

Mak, J., D. P. Marshall, J. R. Maddison, and S. D. Bachman, 2017: Emergent eddy saturation from an energy constrained parameterization. Ocean Modell., 112, 125-138.

Marshall, D. P., and A. J. Adcroft, 2010: Parameterization of ocean eddies: Potential vorticity mixing, energetics and Arnold's first stability theorem. Ocean Modell., 32, 188-204.

Marshall, D. P., J. R. Maddison, and P. S. Berloff, 2012: A framework for parameterizing eddy potential vorticity fluxes. J. Phys. Oceanogr., 42, 539-557, doi:10.1175/JPO-D-11-048.1.

Marshall, J., and K. Speer, 2012: Closure of the meridional overturning circulation through Southern Ocean upwelling. Nat. Geosci., 5, 171-180, doi:10.1038/ngeo1391.

Marston, J. B., 2010: Statistics of the general circulation from cumulant expansions. Chaos, 20, 041 107, doi:10.1063/1.3490719.

Marston, J. B., E. Conover, and T. Schneider, 2008: Statistics of an unstable barotropic jet from a cumulant expansion. J. Atmos. Sci., 65 (6), 1955-1966, doi:10.1175/2007JAS2510.1.

Morrow, R., R. Coleman, J. Church, and D. Chelton, 1994: Surface eddy momentum flux and velocity variances in the Southern Ocean from Geostat altimetry. J. Phys. Oceanogr., 24, 2050 2071.

Parker, J. B., and J. A. Krommes, 2013: Zonal flow as pattern formation. Phys. Plasmas, 20, 100703 , doi:10.1063/1.4828717. 
Parker, J. B., and J. A. Krommes, 2014: Generation of zonal flows through symmetry breaking of statistical homogeneity. New J. Phys., 16 (3), 035 006, doi:10.1088/1367-2630/16/3/035006.

Poulsen, M. B., M. Jochum, J. R. Maddison, D. P. Marshall, and R. Nuterman, 2019: A geometric interpretation of SouthernOcean eddy form stress. J. Phys. Oceanogr., 49, 2553-2570.

Preisendorfer, R. W., 1988: Principal Component Analysis in Meteorology and Oceanography, Developments in Atmospheric Science, Vol. 17. Elsevier, 444 pp.

Qiu, B., 2000: Interannual variability of the Kuroshio Extension system and its impact on the wintertime SST field. J. Phys. Oceanogr., 30, 1486-1502.

Scott, R. B., B. K. Arbic, C. L. Holland, A. Sen, and B. Qiu, 2008: Zonal versus meridional velocity variance in sattelite observations and realistic and idealized ocean circulation models. Ocean Modell., 23, 102-112.

Srinivasan, K., and W. R. Young, 2012: Zonostrophic instability. J. Atmos. Sci., 69 (5), 1633-1656, doi:10.1175/JAS-D-11-0200.1.

Srinivasan, K., and W. R. Young, 2014: Reynold stress and eddy difusivity of $\beta$-plane shear flows. J. Atmos. Sci., 71 (6), 2169-2185, doi:10.1175/JAS-D-13-0246.1.

Stewart, K. D., P. Spence, S. Waterman, J. L. Sommer, J.-M. Molines, J. M. Lilly, and M. H. England, 2015: Anisotropy of eddy variability in the ocean. Ocean Modell., 95, 53-65.

Tamarin, T., J. R. Maddison, E. Heifetz, and D. P. Marshall, 2016: A geometric interpretation of eddy reynolds stresses in barotropic ocean jets. J. Phys. Oceanogr., 46 (8), 2285-2307, doi: 10.1175/JPO-D-15-0139.1, URL https://doi.org/10.1175/JPO-D-15-0139.1.

Tobias, S. M., K. Dagon, and J. B. Marston, 2011: Astrophysical fluid dynamics via direct numerical simulation. Astrophys. J., 727, 127, doi:10.1088/0004-637X/727/2/127. 
Trani, M., P. Falco, and E. Zambianchi, 2011: Near-surface eddy dynamics in the Southern Ocean. Polar Res., 23, 11203.

Vallis, G. K., and M. E. Maltrud, 1993: Generation of mean flows and jets on a beta-plane and over topography. J. Phys. Oceanogr., 23, 1346-1362, doi:10.1175/1520-0485(1993)023<1346: GOMFAJ $\rangle 2.0 . \mathrm{CO} ; 2$.

Walkden, G. J., K. J. Heywood, and D. P. Stevens, 2008: Eddy heat fluxes from direct current measurements of the Antarctic Polar Front in Shag Rocks Passage. J. Geophys. Res., 35, L06 602, doi:10.1029/2007GL032767.

Waterman, S., and B. J. Hoskins, 2013: Eddy shape, orientation, propagation and mean flow feedback in western boundary currents. J. Phys. Oceanogr., 43, 1666-1690.

Waterman, S., and J. M. Lilly, 2015: Geometric decomposition of eddy feedbacks in barotropic systems. J. Phys. Oceanogr., 45, 1009-1024.

Wilkin, J. L., and R. A. Morrow, 1994: Eddy kinetic energy and momentum flux in the Southern Ocean: Comparison of a global eddy-resolving model with altimeter, drifter and current meter data. J. Geophys. Res., 99, 7903-7916.

Youngs, M. K., A. F. Thompson, A. Lazar, and K. J. Richards, 2017: ACC meanders, energy transfer, and mixed barotropic—baroclinic instability. J. Phys. Oceanogr., 47, 1291-1305, doi: 10.1175/JPO-D-16-0160.1. 


\section{LIST OF FIGURES}

Fig. 1. Schematic representation of the eddy variance ellipse in the eddy velocity space. Noted are the angle of the tilt of the ellipse $\theta$, the semi-major axis $a$ (solid), the linear eccentricity $F$ (dashed), and their expressions in terms of the anisotropy parameter $\gamma$ and the eddy kinetic energy $K$.

Fig. 2. Schematic representation of the changes in the eddy variance ellipse with latitude that lead to an eastward acceleration in the flow. In each of the columns one of the parameters $\gamma, \theta$ and $K$ are changing. Column A: $K$ decreasing with $y$ and $\theta$ is in the first (or third) quadrant. Column B: $K$ increasing with $y$ and $\theta$ is in the second (or fourth) quadrant. Column C: $\gamma$ decreasing with $y$ and $\theta$ is in the first (or third) quadrant. Column D: $\gamma$ increasing with $y$ and $\theta$ is in the second (or fourth) quadrant. Column E: $\theta$ decreasing with $y$ and $|\theta|<\pi / 4$. Column F: $\theta$ increasing with $y$ and $|\theta|<\pi / 4$. Figure taken from Tamarin et al. (2016).

Fig. 3. (a)-(b) Amplitude of (a) the momentum fluxes $\hat{N}_{1}$ and (b) the eddy anisotropy $\hat{M}_{1}$ calculated at the onset of zonostrophic instability as a function of $\beta$ for isotropic forcing $(\eta=0)$. (c) Amplitude of the momentum fluxes $\hat{N}_{1}$ calculated at the onset of zonostrophic instability as a function of $\beta$ for anisotropic forcing $(\eta=1)$. Also shown are the asymptotic expressions (A13), (A15) and (A29) (dashed lines).

Fig. 4. (a) Tilt angle $\theta_{1}$ and (b) anisotropy parameter $\gamma_{1}$ as a function of latitude for the most unstable jet perturbation $\bar{u}_{1}=\sin \left(n_{c} y\right)$ and $\beta=0.1$ (solid line) and $\beta=10$ (dashed line). (c) Schematic change of the variance ellipse within the most unstable jet perturbation shown by the thick line. The ellipse eccentricity is of the same order as the infinitesimal amplitude of the mode $(\mathscr{O}(\mu) \ll 1)$, so changes in the ellipse eccentricity are exaggerated by $1 / \mu$ for illustration purposes. The forcing is isotropic $(\eta=0)$.

Fig. 5. The contribution of (a) the anisotropy parameter gradient and (b) the tilt gradient to the acceleration of the most unstable jet perturbation for $\beta=0.1$ (solid line) and $\beta=10$ (dashed line). The forcing is isotropic $(\eta=0)$.

Fig. 6. Schematic change of the variance ellipse within the most unstable jet perturbation shown by the thick line. The ellipse eccentricity is $1-\mathscr{O}(\mu)$, where $\mu \ll 1$ is the infinitesimal amplitude of the jet perturbation. So the ellipse eccentricity is set to 0.99 for illustration purposes. Similarly, changes in the tilt angle are of $\mathscr{O}(\mu)$, so changes in the angle are exaggerated by $1 / \mu$ for illustration purposes. The forcing is anisotropic $(\eta=1)$.

Fig. 7. The non-linear correction of (a) the tilt angle $\theta_{3, N L}$ and (b) the eddy anisotropy parameter $\gamma_{3, N L}$ as a function of latitude (solid lines). The contributions $\theta_{3, e c}, \gamma_{3, e c}$ (dashed line) and $\theta_{3,12}, \gamma_{3,12}$ (dotted line) to the tilt and anisotropy parameter corrections are also shown. (c) The contribution of the anisotropy parameter gradient $f_{d \gamma}$ (solid) and the tilt angle gradient $f_{d \theta}$ (dashed) to the acceleration of the most unstable jet perturbation. The planetary vorticity gradient is $\beta=0.1$ and the forcing is isotropic $(\eta=0)$.

Fig. 8. The same as in Fig. 7 but for $\beta=10$.

Fig. 9. Path of integration to calculate $I^{(\mathrm{NR})}$. Shown is the path $\mathscr{C}_{0}$ (solid line) over which the integral is calculated and the complimentary paths $\mathscr{C}_{j}$ (dashed lines) with $j=1, \ldots, 4$ around the angles $\phi_{j}$ (circles) with the help of which the integral is calculated. The pole of the integrand at $z=0$ is also shown (circle). 


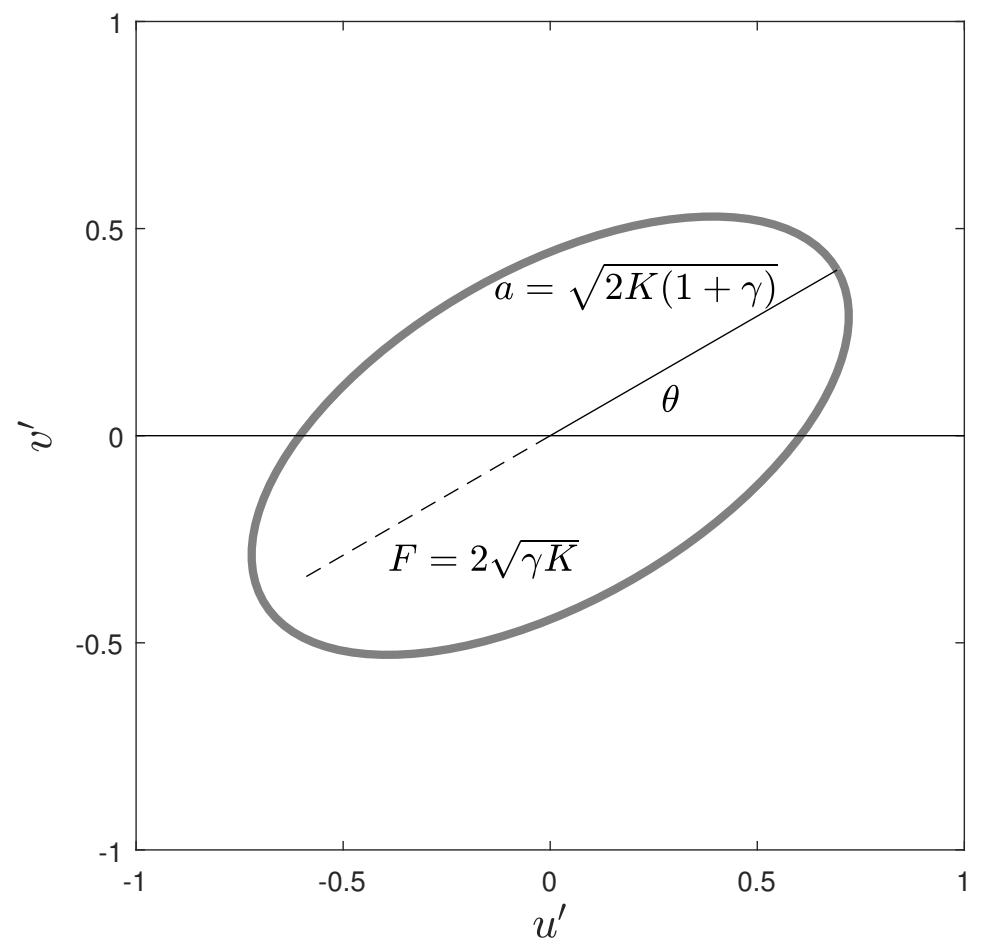

FIG. 1. Schematic representation of the eddy variance ellipse in the eddy velocity space. Noted are the angle of the tilt of the ellipse $\theta$, the semi-major axis $a$ (solid), the linear eccentricity $F$ (dashed), and their expressions in terms of the anisotropy parameter $\gamma$ and the eddy kinetic energy $K$. 


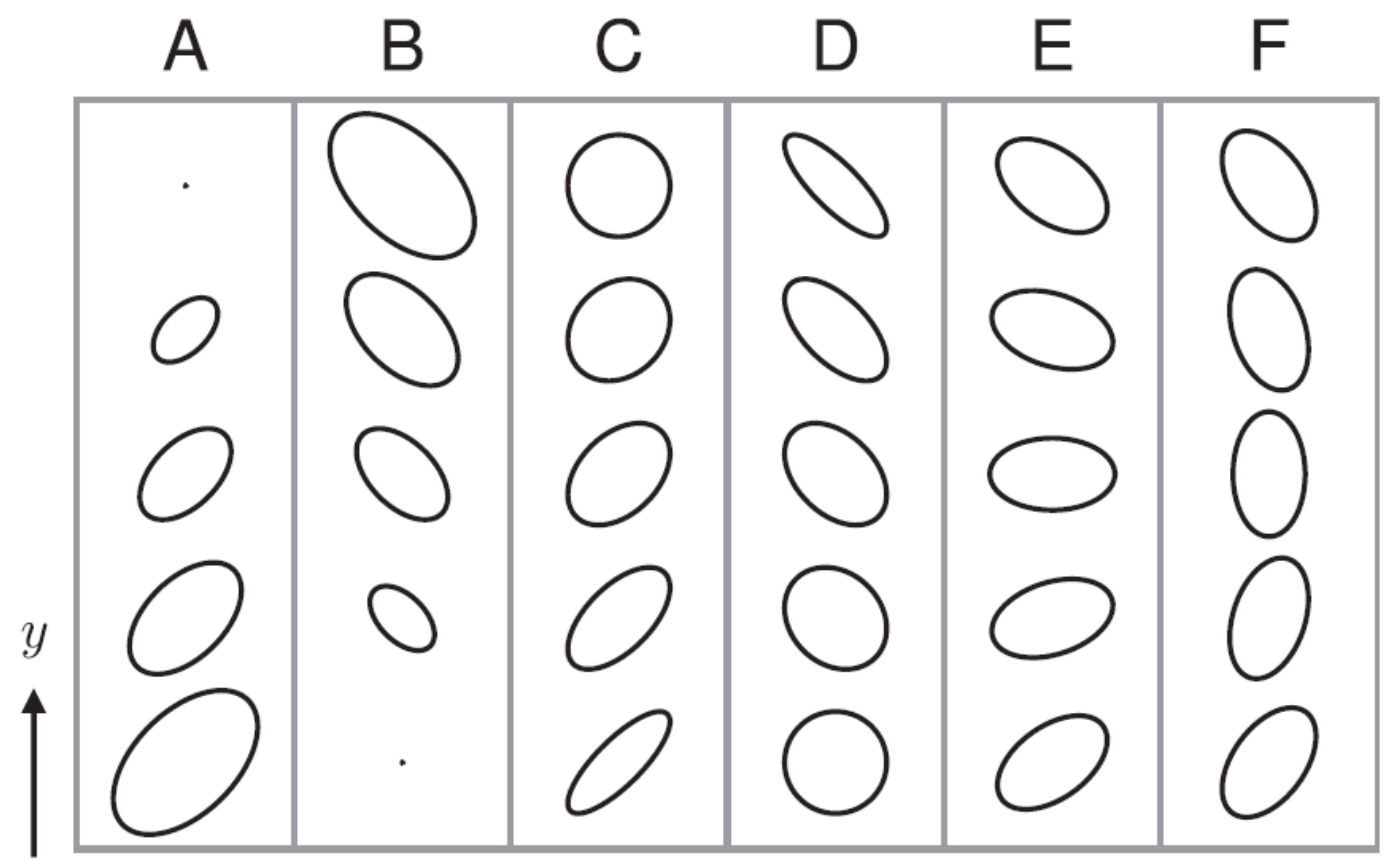

FIG. 2. Schematic representation of the changes in the eddy variance ellipse with latitude that lead to an eastward acceleration in the flow. In each of the columns one of the parameters $\gamma, \theta$ and $K$ are changing. Column A: $K$ decreasing with $y$ and $\theta$ is in the first (or third) quadrant. Column B: $K$ increasing with $y$ and $\theta$ is in the second (or fourth) quadrant. Column C: $\gamma$ decreasing with $y$ and $\theta$ is in the first (or third) quadrant. Column D: $\gamma$ increasing with $y$ and $\theta$ is in the second (or fourth) quadrant. Column E: $\theta$ decreasing with $y$ and $|\theta|<\pi / 4$. Column F: $\theta$ increasing with $y$ and $|\theta|<\pi / 4$. Figure taken from Tamarin et al. (2016). 
(a)

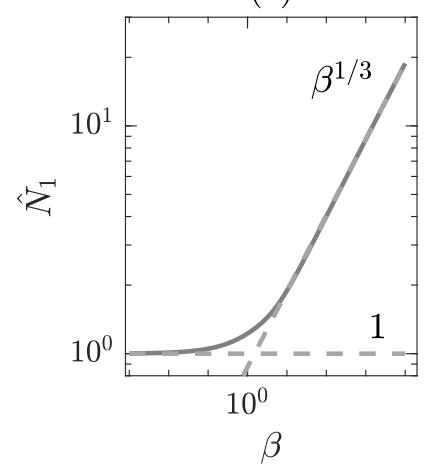

(b)

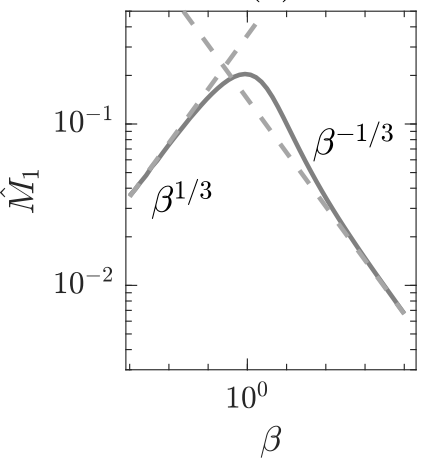

(c)

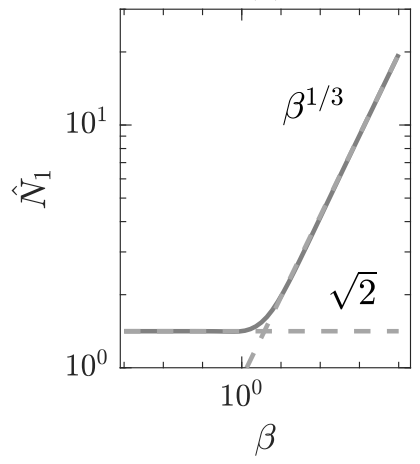

FIG. 3. (a)-(b) Amplitude of (a) the momentum fluxes $\hat{N}_{1}$ and (b) the eddy anisotropy $\hat{M}_{1}$ calculated at the onset of zonostrophic instability as a function of $\beta$ for isotropic forcing $(\eta=0)$. (c) Amplitude of the momentum fluxes $\hat{N}_{1}$ calculated at the onset of zonostrophic instability as a function of $\beta$ for anisotropic forcing $(\eta=1)$. Also shown are the asymptotic expressions (A13), (A15) and (A29) (dashed lines). 
(a)

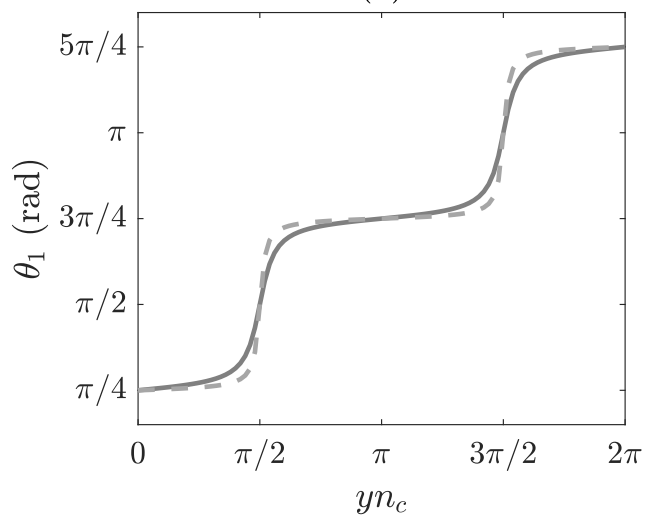

(b)

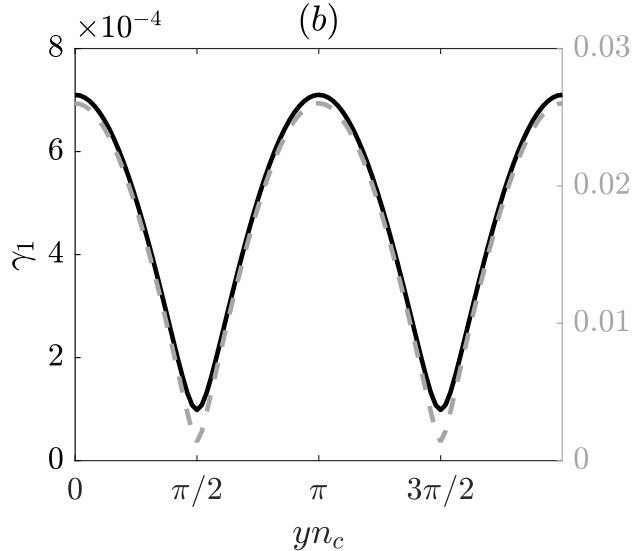

$(c)$

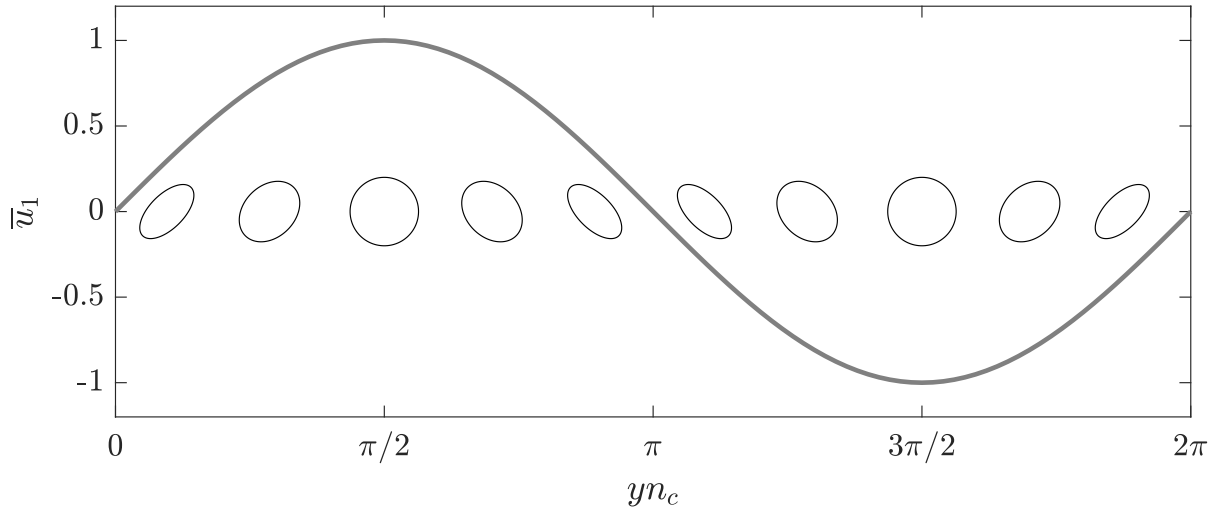

FIG. 4. (a) Tilt angle $\theta_{1}$ and (b) anisotropy parameter $\gamma_{1}$ as a function of latitude for the most unstable jet perturbation $\bar{u}_{1}=\sin \left(n_{c} y\right)$ and $\beta=0.1$ (solid line) and $\beta=10$ (dashed line). (c) Schematic change of the variance ellipse within the most unstable jet perturbation shown by the thick line. The ellipse eccentricity is of the same order as the infinitesimal amplitude of the mode $(\mathscr{O}(\mu) \ll 1)$, so changes in the ellipse eccentricity are exaggerated by $1 / \mu$ for illustration purposes. The forcing is isotropic $(\eta=0)$. 
(a)

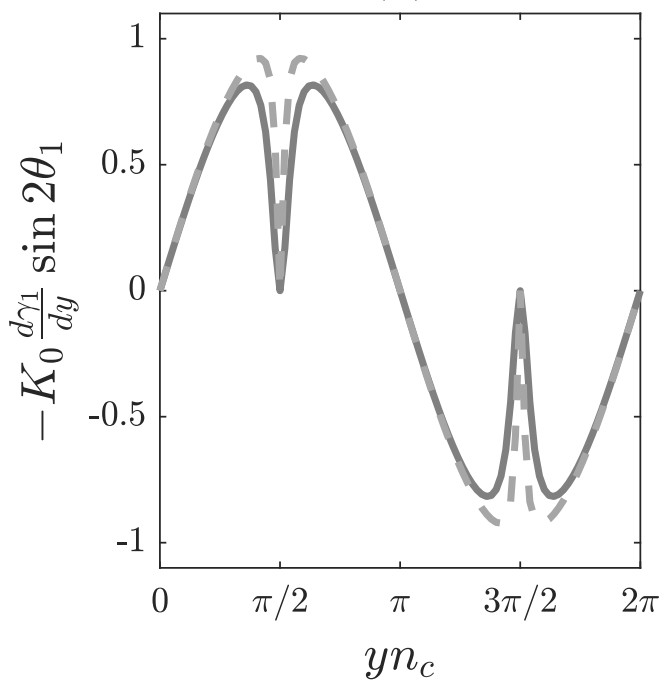

(b)

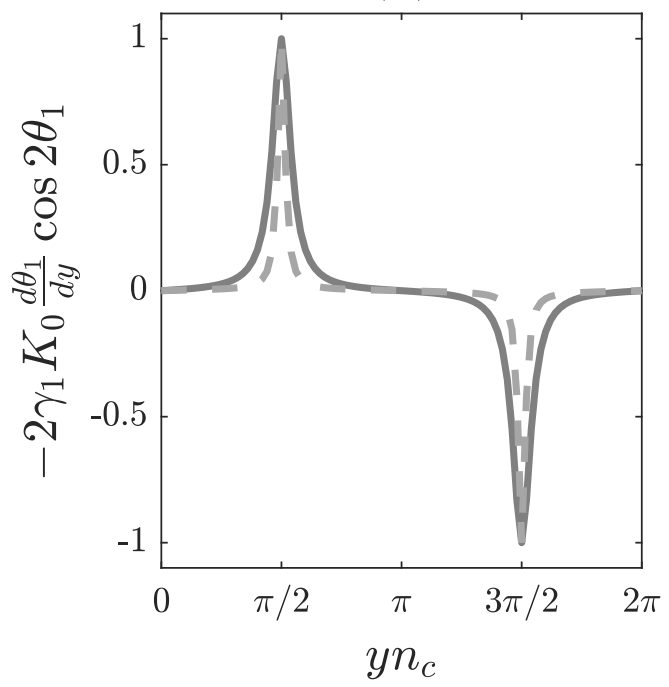

FIG. 5. The contribution of (a) the anisotropy parameter gradient and (b) the tilt gradient to the acceleration of the most unstable jet perturbation for $\beta=0.1$ (solid line) and $\beta=10$ (dashed line). The forcing is isotropic $(\eta=0)$. 


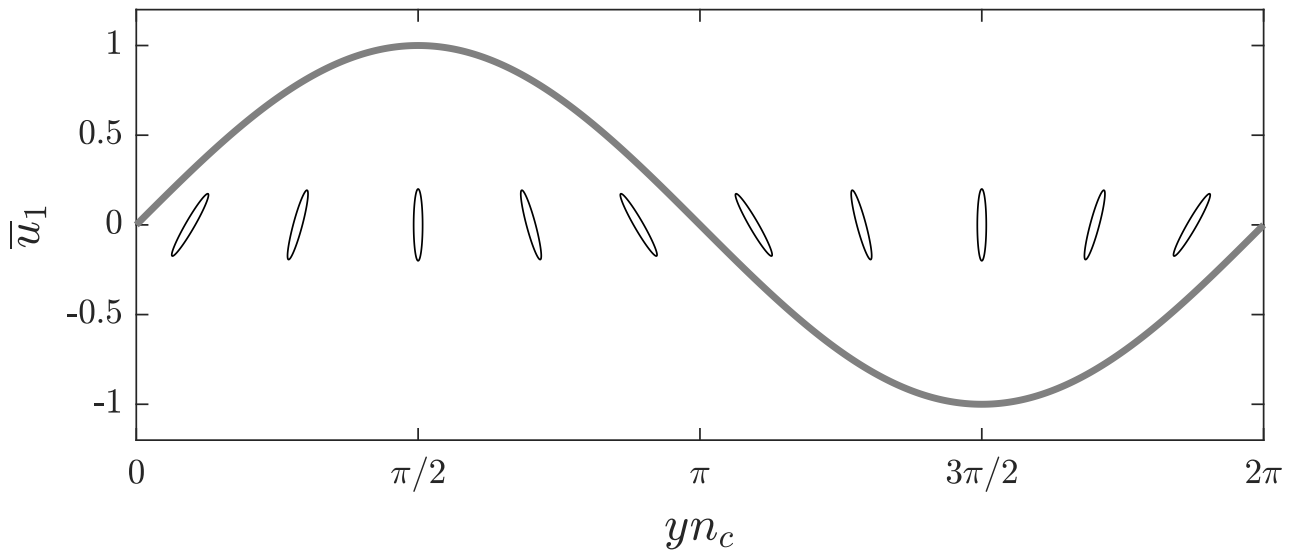

FIG. 6. Schematic change of the variance ellipse within the most unstable jet perturbation shown by the thick line. The ellipse eccentricity is $1-\mathscr{O}(\mu)$, where $\mu \ll 1$ is the infinitesimal amplitude of the jet perturbation. So the ellipse eccentricity is set to 0.99 for illustration purposes. Similarly, changes in the tilt angle are of $\mathscr{O}(\mu)$, so changes in the angle are exaggerated by $1 / \mu$ for illustration purposes. The forcing is anisotropic $(\eta=1)$. 

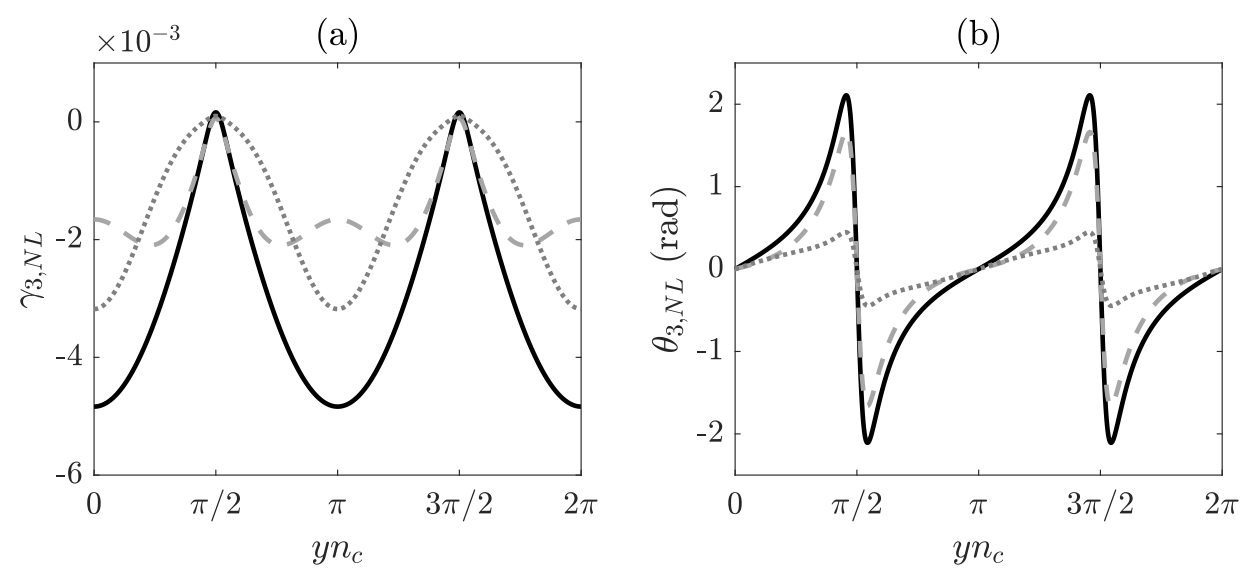

(c)

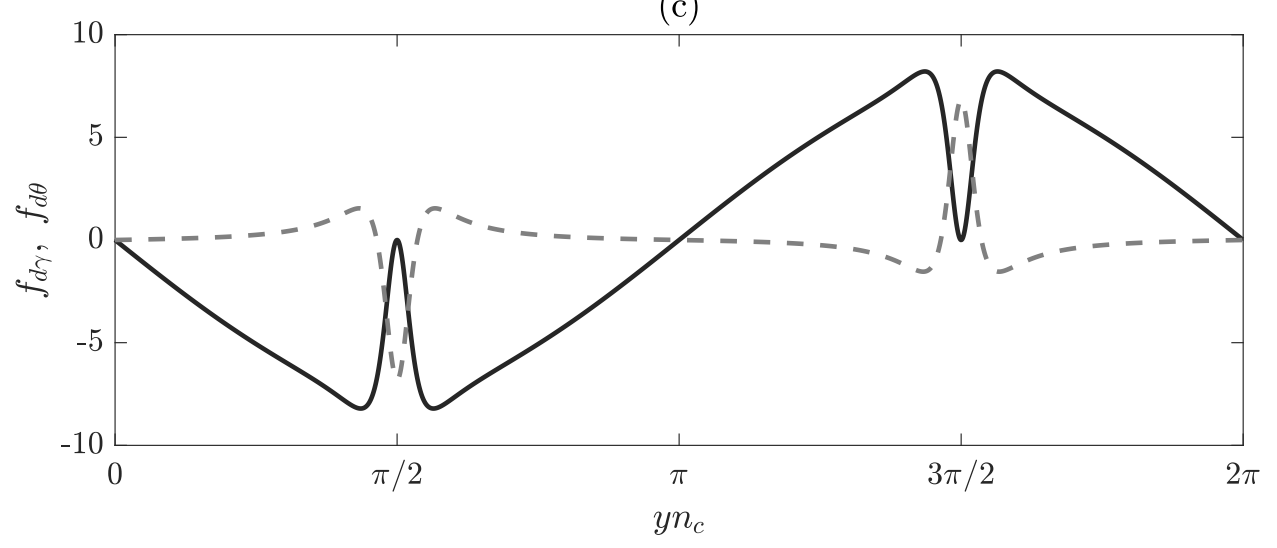

FIG. 7. The non-linear correction of (a) the tilt angle $\theta_{3, N L}$ and (b) the eddy anisotropy parameter $\gamma_{3, N L}$ as a function of latitude (solid lines). The contributions $\theta_{3, e c}, \gamma_{3, e c}$ (dashed line) and $\theta_{3,12}, \gamma_{3,12}$ (dotted line) to the tilt and anisotropy parameter corrections are also shown. (c) The contribution of the anisotropy parameter gradient $f_{d \gamma}$ (solid) and the tilt angle gradient $f_{d \theta}$ (dashed) to the acceleration of the most unstable jet perturbation. The planetary vorticity gradient is $\beta=0.1$ and the forcing is isotropic $(\eta=0)$. 

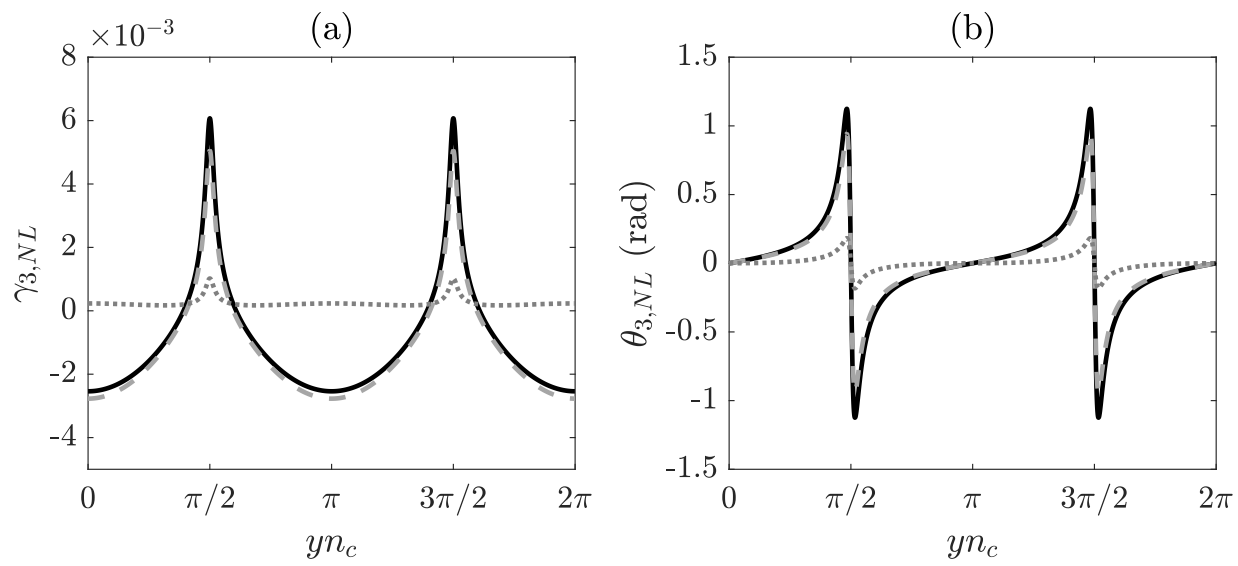

(c)

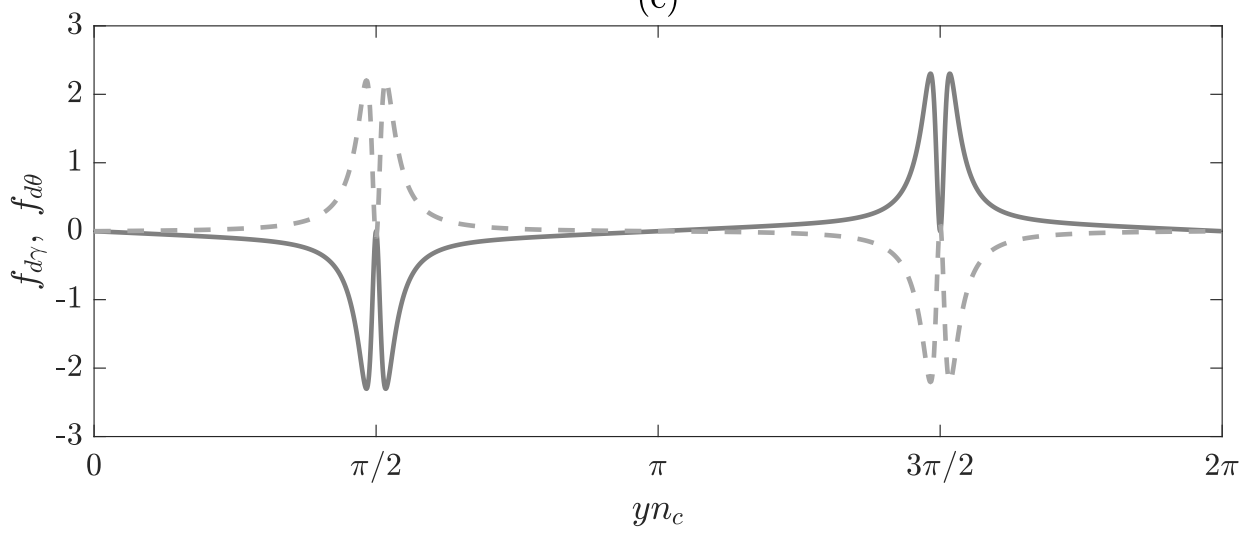

FIG. 8. The same as in Fig. 7 but for $\beta=10$. 


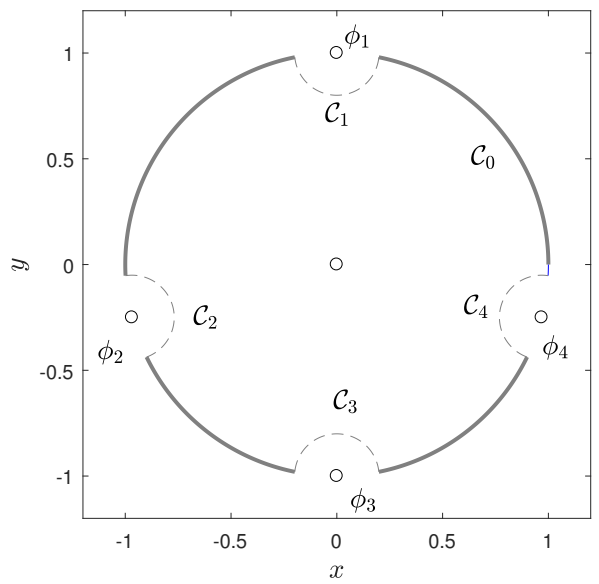

FIG. 9. Path of integration to calculate $I^{(\mathrm{NR})}$. Shown is the path $\mathscr{C}_{0}$ (solid line) over which the integral is calculated and the complimentary paths $\mathscr{C}_{j}$ (dashed lines) with $j=1, \ldots, 4$ around the angles $\phi_{j}$ (circles) with the help of which the integral is calculated. The pole of the integrand at $z=0$ is also shown (circle). 